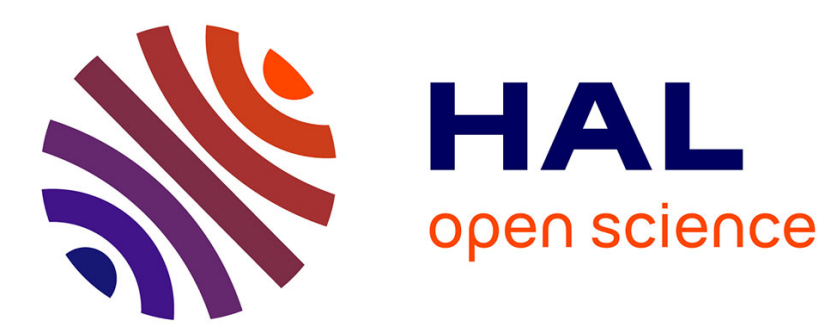

\title{
Fitting spatial max-mixture processes with unknown extremal dependence class: an exploratory analysis tool
}

Abdul-Fattah Abu-Awwad, Véronique Maume-Deschamps, Pierre Ribereau

\section{To cite this version:}

Abdul-Fattah Abu-Awwad, Véronique Maume-Deschamps, Pierre Ribereau. Fitting spatial maxmixture processes with unknown extremal dependence class: an exploratory analysis tool. Test, 2020, $29,10.1007 / \mathrm{s} 11749-019-00663-5$. hal-01671380

\section{HAL Id: hal-01671380 \\ https://hal.science/hal-01671380}

Submitted on 2 Jan 2018

HAL is a multi-disciplinary open access archive for the deposit and dissemination of scientific research documents, whether they are published or not. The documents may come from teaching and research institutions in France or abroad, or from public or private research centers.
L'archive ouverte pluridisciplinaire HAL, est destinée au dépôt et à la diffusion de documents scientifiques de niveau recherche, publiés ou non, émanant des établissements d'enseignement et de recherche français ou étrangers, des laboratoires publics ou privés. 


\title{
A MODEL-FREE SELECTION CRITERION FOR THE MIXING COEFFICIENT OF SPATIAL MAX-MIXTURE MODELS
}

\author{
ABU-AWWAD ABDUL-FATTAH, MAUME-DESCHAMPS VÉRONIQUE, AND RIBEREAU PIERRE
}

\begin{abstract}
One of the main concerns in extreme value theory is to quantify the dependence between joint tails. Using stochastic processes that lack flexibility in the joint tail may lead to severe under- or over-estimation of probabilities associated to simultaneous extreme events. Following recent advances in the literature, a flexible model called max-mixture model has been introduced for modeling situations where the extremal dependence structure may vary with the distance. In this paper we propose a nonparametric model-free selection criterion for the mixing coefficient Our criterion is derived from a madogram, a notion classically used in geostatistics to capture spatial structures. The procedure is based on a nonlinear least squares between the theoretical madogram and the empirical one. We perform a simulation study and apply our criterion to daily precipitation over the East of Australia.
\end{abstract}

Keywords: Extremal dependence; Madogram; Max-stable model; Max-mixture model; Nonlinear least squares.

\section{INTRODUCTION}

Max-stable stochastic processes arise as a fundamental class of models that are able to describe spatial extreme value phenomena. Max-stable process models for spatial data were first constructed using the spectral representation (De Haan, 1984). Several subsequent works on the construction of spatial max-stable processes have been developed, see e.g. (Smith, 1990; Schlather, 2002; Kabluchko et al., 2009; Davison and Gholamrezaee, 2012). The inference on spatial processes is an open field that is still in development. Both parametric and nonparametric inference methods are used in the literature.

Camille Jordan Institute - ICJ, Université Claude Bernard Lyon 1, France

Address for correspondence: A. Abu-Awwad, Department of Applied Mathematics, School of informatics and mathematics, Camille Jordan Institute - ICJ, Université Claude Bernard Lyon 1, 43 Boulevard du 11 novembre 1918, F-69622 Villeurbanne Cedex, France.

E-mail addresses: abuawwad@math.univ-lyon1.fr, veronique.maume@univ-lyon1.fr, pierre.ribereau@univ-lyon1.fr. 
For a stationary spatial process $X=\{X(s), s \in \mathcal{S}\}$, the Asymptotic Dependence (AD) is characterized by $\chi(h)>0$, with:

$$
\chi(h)=\lim _{x \rightarrow \infty} \mathbb{P}\{X(s)>x \mid X(s+h)>x\}, \quad s, s+h \in \mathcal{S} .
$$

This means that, for an $\mathrm{AD}$ process, a large event at location $s+h$ leads to a non-zero probability of a similarly large event at location $s$ for some spatial lag vector $h$. On one other hand, a process is Asymptotically Independent (AI) if $\chi(h)=0$ for any $h$. This is achieved e.g. for Gaussian processes, see (Sibuya, 1960).

Within the class of max-stable models, only two types of dependence structures are possible: either the process is $\mathrm{AD}$ or it is independent. This restriction leads to a drawback of max-stable processes that they are too coarse to describe multivariate joint tails with asymptotic independence sufficiently accurately. Particularly, fitting asymptotic dependent models to asymptotically independent data leads over/under estimation of probabilities of extreme joint events, since there is a mis-placed assumption that the most extreme marginal events may occur simultaneously (Coles et al., 1999). (Thibaud et al., 2013; Davison et al., 2013) introduced recent examples about practical difficulties to identify whether a data set should be modeled using an asymptotically dependent or asymptotically independent.

(Wadsworth and Tawn, 2012) introduced a new class of models, so-called max-mixture models, to capture both AD and AI. The basic idea is to mix max-stable and asymptotic independent processes. Let $a \in[0,1]$, then the max-mixture (MM) model is defined as

$$
Z(s)=\max \{a X(s),(1-a) Y(s)\}, \quad s \in \mathcal{S}
$$

where $X(s)$ is a stationary max-stable process and $Y(s)$ is a stationary AI process, so that the parameter $a$ represents the proportion of $\mathrm{AD}$ in the process $Z$.

In this paper, we are concerned with constructing a model-free criterion to choose a realistic value for the mixing parameter $a$. Our objective is not to model extremal dependence of joint tails but to set up a statistical criterion that facilitate the modelling of the spatial data with suitable behaviour. We shall use least squares on the $F^{\lambda}$-madogram. In (Bel et al., 2008), a madogram based test on the $\mathrm{AD}$ of a max-stable process is proposed, while in (Abu-Awwad 
et al., 2017), a parametric test on $a$ for max-mixture processes is developed.

The paper is organized as follows. Section 2 reviews spatial extremes processes. The proposed $F^{\lambda}$-madogram for max-mixture models and the selection criterion for the mixing coefficient $a$ are developed in Section 3, while Section 4 illustrates the performance of our method through a number of simulation studies. We conclude with an illustration of spatial analysis of precipitation in Australia in Section 5.

\section{Spatial eXtremes PROCESSES: MOdels}

Throughout our work, $X=\{X(s), s \in \mathcal{S}\}, \mathcal{S} \subset \mathbb{R}^{d}$ (generally, $d=2$ ) is a spatial process, it will be assumed to be stationary and isotropic.

2.1. Max-stable processes. Suppose that $\left\{Y_{i}(s): s \in \mathcal{S} \subset \mathbb{R}^{d}\right\}, i=0,1,2$, .., are i.i.d replicates of a random process $Y(s)$, and that there are sequences of continuous functions $\left\{a_{n}(s)>0\right\}$ and $\left\{b_{n}(s)\right\}$ such that, the rescaled process of maxima,

$$
\bigvee_{i=1}^{n} \frac{Y_{i}(s)-b_{n}(s)}{a_{n}(s)} \stackrel{d}{\rightarrow} X(s), \quad n \rightarrow \infty
$$

where the limiting random process $X$ is assumed to be non-degenerate. By (De Haan and Pereira, 2006) the class of the limiting processes $X(s)$ coincides with the class of max-stable processes. This definition of MS processes offers a natural choice for modeling spatial extremes.

The univariate extreme value theory, implies that the marginal distributions of $X(s)$ are Generalized Extreme value (GEV) distributed, and without loss of generality the margins can transformed to a simple MS process called standard Fréchet distribution, $\mathbb{P}(X(s) \leq z)=\exp \left\{-z^{-1}\right\}$.

Following (De Haan, 1984; Schlather, 2002), a simple MS process $X(s)$ has the following representation

$$
X(s)=\max _{k \geq 1} Q_{k}(s) / P_{k}, \quad s \in \mathcal{S} .
$$


where $Q_{k}(s)$ are independent replicates of a non-negative stochastic process $Q(s)$ with unit mean at each $s$, and $P_{k}$ are the points of a unit rate Poisson process $(0, \infty)$.

For $K \in \mathbb{N} \backslash\{0\}, s_{1}, \ldots, s_{K} \in \mathcal{S}$, and $x_{1}, \ldots, x_{K}>0$, the finite $K$-dimensional distribution of the process $X$ owing to the representation( 2.2) is given by

$$
-\log \mathbb{P}\left(X\left(s_{1}\right) \leq z_{1}, \ldots, X\left(s_{K}\right) \leq z_{K}\right)=\mathbb{E}\left[\bigvee_{k=1}^{K}\left\{\frac{Q\left(s_{k}\right)}{z_{k}}\right\}\right]=V_{s_{1}, \ldots, s_{K}}\left(z_{1}, \ldots, z_{K}\right)
$$

where $V_{s_{1}, \ldots, s_{K}}($.$) is called the exponent measure. It summarises the structure of extremal$ dependence, and satisfies the property of homogenity of order -1 and $V_{s_{1}, \ldots, s_{K}}(\infty, \ldots, z, \ldots, \infty)=$ $z^{-1}$. It has to be noted that

$$
-z \log \mathbb{P}\left\{X\left(s_{1}\right) \leq z, . ., X\left(s_{K}\right) \leq z\right\}=V_{s_{1}, \ldots, s_{K}}(1, \ldots, 1)=\theta_{s_{1}, \ldots, s_{K}}
$$

The coefficient $\theta_{s_{1}, \ldots, s_{K}}$ is known as the extremal coefficient. It can be seen as a summary of extremal dependence with two boundary values. The complete independence is achieved when $\theta_{s_{1}, \ldots, s_{K}}=1$, while complete independence is achieved when $\theta_{s_{1}, \ldots, s_{K}}=K$. In the bivariate case, the $\mathrm{AI}$ and $\mathrm{AD}$ between a pair of random variables $Z_{1}$ and $Z_{2}$, with marginal distributions $F_{1}$ and $F_{2}$, may be identified by

$$
\left.\chi=\lim _{u \rightarrow 1^{-}} P\left(F_{1}\left(Z_{1}\right)>u \mid F_{2}\left(Z_{2}\right)>u\right)\right) .
$$

The cases $\chi=0$ and $\chi>0$ represent $\mathrm{AI}$ and AD, respectively, (Joe, 1993). This coefficient is related to the pairwise extremal coefficient $\theta$ through the relation $\chi=2-\theta$.

Since both dependence functions $\theta$ and $\chi$ are useless for AI processes, (Coles et al., 1999) proposed a new dependence coefficient which measures the strength of dependence for AI processes:

$$
\bar{\chi}=\lim _{u \rightarrow 1^{-}} \bar{\chi}(u)=\lim _{u \rightarrow 1^{-}} \frac{2 \log P(F(Z(s))>u)}{\log P(F(Z(s))>u, F(Z(s+h))>u)}-1
$$

$\mathrm{AD}$ (respectively $\mathrm{AI})$ is achieved if and if $\bar{\chi}=1$ (resp. $\bar{\chi}<1)$.

Another dependence model for bivariate joint tails was introduced by (Ledford and Tawn, 1996)

$$
\mathbb{P}(X(s)>x, X(s+h)>x) \sim x^{-1 / \eta(h)} \mathcal{L}_{h}(x), \text { for } \quad x \rightarrow \infty
$$


where $X$ is a stationary spatial process with unit Fréchet margins, $\mathcal{L}_{h}($.$) is a slowly varying$ function at $\infty$ and the tail dependence coefficient $\eta(h) \in(0,1]$. AI corresponds to $\eta(h)<1$.

Different choices for the process $Q(s)$ in (2.2) lead to more or less flexible models for spatial maxima. Commonly used models are the Guassian extreme value process (Smith, 1990), the extremal Gaussian process (Schlather, 2002), the Brown-Resnick process (Kabluchko et al., 2009), and the extremal-t process (Opitz, 2013). Below, we list these four specific examples of max-stable models.

The storm profile model (Smith, 1990), is defined by taking $Q_{k}(s)=f\left(s-W_{k}\right)$ in Equation (2.2), where $f$ is the density function of a Gaussian random vector with covariance matrix $\Sigma \in \mathbb{R}^{2 \times 2}$. The function $f$ plays a major role as it determines the shape of the storm events. $W_{k}$ is a homogenous Poisson process. The bivariate exponent function of the Smith model has the form

$$
\begin{aligned}
-\log \mathbb{P} & {\left[X(s) \leq x_{1}, X(s+h) \leq x_{2}\right] } \\
& =\frac{1}{x_{1}} \Phi\left(\frac{\beta(h)}{2}+\frac{1}{\beta(h)} \log \left(\frac{x_{2}}{x_{1}}\right)\right)+\frac{1}{x_{2}} \Phi\left(\frac{\beta(h)}{2}+\frac{1}{\beta(h)} \log \left(\frac{x_{1}}{x_{2}}\right)\right)
\end{aligned}
$$

where $\beta(h)=\sqrt{h^{T} \Sigma^{-1} h}$ and $\Phi$ is the standard normal distribution function. In this case the extremal coefficient is equal to $\theta(h)=2 \Phi\{\beta(h) / 2\}$.

A model originally due to (Schlather, 2002) is the Truncated Extremal Gaussian (TEG) model and has been exemplified in (Davison and Gholamrezaee, 2012). This process is obtained by taking $Q_{k}(s)=c \max \left(0, \varepsilon_{k}(s)\right) \mathbb{1}_{\mathcal{A}_{k}}\left(s-R_{k}\right)$, where $\varepsilon_{k}(s)$ are independent replicates of a stationary Gaussian process $\varepsilon=\{\varepsilon(s), s \in \mathcal{S}\}$ with zero mean, unit variance and correlation function $\rho($.$) . \mathbb{1}_{\mathcal{A}}$ is the indicator function of a compact random set $\mathcal{A} \subset \mathcal{S}, \mathcal{A}_{k}$ are independent replicates of $\mathcal{A}$ and $R_{k}$ are points of a Poisson process with a unit rate on $\mathcal{S}$. The constant $c$ is chosen to satisfy the constraint $\mathbb{E}\left\{Q_{k}(s)\right\}=1$.

The bivariate exponent function of a TEG model in the stationary case has the form

$$
-\log \mathbb{P}\left[X(s) \leq x_{1}, X(s+h) \leq x_{2}\right]=\left(\frac{1}{x_{1}}+\frac{1}{x_{2}}\right)\left[1-\frac{\alpha(h)}{2}\left(1-\sqrt{1-\frac{2(\rho(h)+1) x_{1} x_{2}}{\left(x_{1}+x_{2}\right)^{2}}}\right)\right]
$$


where $\alpha(h)=(1-h / 2 r) \mathbb{1}_{[0,2 r]}$ if $\mathcal{A}$ is a disk of fixed radius $r$. This yields $\theta(h)=2-\alpha(h)\{1-$ $\sqrt{(1-\rho(h)) / 2}\}$.

The max-stable Brown-Resnick (BR) process model proposed by (Brown and Resnick, 1977; Kabluchko et al., 2009) is a stationary max-stable process that can be constructed with $Q_{k}(s)=$ $\exp \left\{\varepsilon_{k}(s)-\gamma(s)\right\}, s \in \mathcal{S}$, where $\varepsilon_{k}(s)$ denotes a Gaussian process with semivariogram $\gamma(h)$. The bivariate exponent function of a BR process is:

$$
\begin{aligned}
-\log \mathbb{P} & {\left[X(s) \leq x_{1}, X(s+h) \leq x_{2}\right] } \\
& =\frac{1}{x_{1}} \Phi\left(\sqrt{\frac{\gamma(h)}{2}}+\frac{1}{\sqrt{2 \gamma(h)}} \log \left(\frac{x_{2}}{x_{1}}\right)\right)+\frac{1}{x_{2}} \Phi\left(\sqrt{\frac{\gamma(h)}{2}}+\frac{1}{\sqrt{2 \gamma(h)}} \log \left(\frac{x_{1}}{x_{2}}\right)\right)
\end{aligned}
$$

where $\gamma$ and $\Phi$ denote respectively the semivariogram and the standard normal distribution function. In particular, when the variogram $2 \gamma(h)=h^{T} \Sigma^{-1} h$ for some covariance matrix $\Sigma$, we recover the bivariate distribution function of a Smith model.The pairwise extremal coefficient for a Brown-Resnick process is $\theta(h)=2 \Phi\{\sqrt{\gamma(h) / 2}\}$.

The extremal- $t$ max-stable process proposed in (Opitz, 2013; Ribatet and Sedki, 2013) can be constructed by using $Q_{k}(s)=\left\{m_{v}^{-1 / v} T_{k}(s)\right\}^{v}$, where $T_{k}$ is a zero mean Gaussian process with correlation function $\rho, v \geq 1,1 / m_{v}=\sqrt{\pi} 2^{v / 2-1} \Gamma\left(\frac{v+1}{2}\right), \Gamma($.$) is the gamma function. This$ process has the bivariate exponent function:

$$
\begin{aligned}
& -\log \mathbb{P}\left[X(s) \leq x_{1}, X(s+h) \leq x_{2}\right] \\
& \quad=\frac{1}{x_{1}} T_{v+1}\left(\alpha \rho(h)+\alpha\left(\frac{x_{2}}{x_{1}}\right)^{1 / v}\right)+\frac{1}{x_{2}} T_{v+1}\left(\alpha \rho(h)+\alpha\left(\frac{x_{1}}{x_{2}}\right)^{1 / v}\right)
\end{aligned}
$$

where $T_{v}$ is the distribution function of a Student random variable with $v$ degrees of freedom and $\alpha=\left[v+1 /\left\{1-\rho^{2}(h)\right\}\right]^{1 / 2}$. For an extremal-t process the degrees of freedom $v$ controls the upper bound of the extremal coefficient: $\theta(h)=2 T_{v+1}\left(\sqrt{(v+1) \frac{1-\rho(h)}{1+\rho(h)}}\right)$.

In this paper, we shall make intensive use of the so-called $F$-madogram that is based on a classical geostatistical tool, the madogram (Matheron, 1987). It has been introduced in (Cooley 
et al., 2006). Let $X$ is a stationary max-stable random process. The marginal distribution function is denoted by $F$. The $F$-madogram is defined by:

$$
\nu_{F}(h)=\frac{1}{2} \mathbb{E}[|F(X(s))-F(X(s+h))|], \quad 0 \leq \nu_{F}(h) \leq 1 / 6 .
$$

The bounds of the $F$ madogram correspond respectively to complete dependence and independence. Due to the one-to-one relationship between the extremal dependence function and the $F$-madogram, a simple estimator for $\theta(h)$ can be derived:

$$
\widehat{\theta}(h)=\frac{0.5+\widehat{\nu}_{F}(h)}{0.5-\widehat{\nu}_{F}(h)}
$$

where $\widehat{\nu}(h)=\frac{1}{2 N} \sum_{i=1}^{N}\left|\widehat{F}\left\{x_{i}(s)\right\}-\widehat{F}\left\{x_{i}(s+h)\right\}\right|, x_{i}(s)$ and $x_{i}(s+h)$ are the $i$-th observations of the random field at locations $s$ and $s+h . \widehat{F}$ is the empirical distribution function, i.e, $\widehat{F}(z)=(N+1)^{-1} \sum_{i=1}^{N} \mathbb{I}_{Z^{i}\left(s_{j}\right) \leq z}$, where $\mathbb{I}($.$) is the indicator function.$

The so-called $F^{\lambda}$-madogram has been introduced for max-stable models by (Bel et al., 2008) as a generalization of $F$-madogram (2.8): for any $\lambda>0$, let

$$
\nu_{F^{\lambda}}(h)=\frac{1}{2} \mathbb{E}\left[\left|F^{\lambda}\{X(s)\}-F^{\lambda}\{X(s+h)\}\right|\right] .
$$

A nonlinear least squares procedure has been proposed by (Bel et al., 2008) based on $F^{\lambda}$-madogram to compute an estimator for the extremal dependence function that may outperforms other known estimators. In that work, it has been found by some trials that good estimations are obtained for $\lambda \in[2,3]$.

2.2. Hybrid models of spatial extremal dependence. Although max-stable models seem to be suitable for modeling extremely high threshold exceedances, asymptotic independence models may show a better fit at finite thresholds. Due to difficulty or impossibility in practice to decide whether a dataset should be modeled using AD or AI, (Wadsworth and Tawn, 2012) have been introduced the hybrid spatial dependence models which are able to capture both AD and AI. 
Consider $Y^{\prime}$ a stationary Gaussian process. Let $Y(s)=-1 / \log \left(\Phi\left(Y^{\prime}(s)\right)\right)$ then, $Y^{\prime}$ is an AI process with unit Frchet marginal distributions. Another class of AI processes called inverted max-stable processes has been proposed by (Wadsworth and Tawn, 2012). They are defined as

$$
Y(s)=-1 / \log \left\{1-\exp \left[-Y^{\prime}(s)^{-1}\right]\right\}
$$

where $Y^{\prime}$ is a simple max-stable process with extremal coefficient $\theta_{Y^{\prime}}$. We a slight abuse of language, we shall denote $\theta_{Y^{\prime}}$ by $\theta_{Y}$. With this construction, each max-stable process may be transformed into an AI independent counterpart. This inverted max-stable process (IMS) satisfies (2.6) and $\eta(h)=1 / \theta_{Y}(h)$. The bivariate distribution function is given by

$$
\mathbb{P}\left(Y(s) \leq y_{1}, Y(s+h) \leq y_{2}\right)=-1+\exp \left(-y_{1}^{-1}\right)+\exp \left(-y_{2}^{-1}\right)+\exp \left\{-V_{Y}\left[\omega\left(y_{1}\right), \omega\left(y_{2}\right)\right]\right\}
$$

where $V_{Y}$ is the exponent measure of the bivariate extreme-distribution of $\left\{Y^{\prime}(s), Y^{\prime}(s+h)\right\}$, and $\omega(y)=-1 / \log \left\{1-\exp \left[-y^{-1}\right]\right\}$. We a slight abuse of language, we shall say that $V_{Y}$ is the exponent measure of $Y$.

We are now in position to define the max-mixture processes that we will be working on. Let $X$ be a simple max-stable process with bivariate extremal coefficient $\theta_{X}$, and $Y$ be an inverted max-stable process with coefficient of tail dependence $\eta$. Assume that $X$ and $Y$ are independent. Then for a mixture proportion $a \in[0,1]$, the spatial max-mixture process proposed by (Wadsworth and Tawn, 2012) is defined as

$$
Z(s)=\max \{a X(s),(1-a) Y(s)\}
$$

Clearly, models that are only $\mathrm{AD}$ or $\mathrm{AI}$ are submodels of $Z$, obtained for $a=1, a=0$, respectively. The bivariate joint survivor function of the process $Z$ satisfies

$$
\mathbb{P}[Z(s)>z, Z(s+h)>z]=\frac{a(2-\theta(h))}{z}+\left(\frac{1-a}{z}\right)^{1 / \eta(h)}+O\left(z^{-2}\right) \quad \text { as } \quad z \rightarrow \infty
$$


If $h_{0}=\inf \{h: \theta(h)=2\}$ is finite, then the process $Z$ is $\mathrm{AD}$ up to distance $h_{0}$, and AI for longer distances. The bivariate CDF for a pair of sites $(Z(s), Z(s+h))$ is straightforwardly obtained by the independence between $X(s), Y(s)$

$\mathbb{P}\left(Z(s) \leq z_{1}, Z(s+h) \leq z_{2}\right)=\mathbb{P}\left(X(s) \leq \frac{z_{1}}{a}, X(s+h) \leq \frac{z_{2}}{a}\right) \mathbb{P}\left(Y(s) \leq \frac{z_{1}}{1-a}, Y(s+h) \leq \frac{z_{2}}{1-a}\right)$

Thus, in the case where $X(s)$ is a max-stable process and $Y(s)$ is a inverted max-stable process, the distribution function in (2.14) has the form

$$
\begin{array}{r}
\mathbb{P}\left(Z(s) \leq z_{1}, Z(s+h) \leq z_{2}\right)=\exp \left\{-a V_{X}\left(z_{1}, z_{2}\right)\right\} \times\left\{-1+\exp \left[(a-1) / z_{1}\right]\right. \\
+\exp \left[(a-1) / z_{2}\right]+\exp \left[-V_{Y}\left[\omega\left((1-a) / z_{1}\right), \omega\left((1-a) / z_{2}\right)\right]\right\}
\end{array}
$$

where $V_{X}$ and $V_{Y}$ are the bivariate exponent measures for $X$ and $Y$ respectively.

Figure 1 displays two simulated images of AI processes over the $[0,10]^{2}$ square. The corresponding functions $\eta(h)$ are also represented. According to (Ledford and Tawn, 1997), the case $\eta(h)=1 / 2$ corresponds to the near-independence, the AI process constructed from a BrownResnick process (this case corresponds to the isotropic Smith process) allows asymptotic independence but tends to near-indpendence for long distances. While an AI process constructed from extremal- $t$ process presents a stronger dependence in the asymptotic independence when $h$ is sufficiently large.
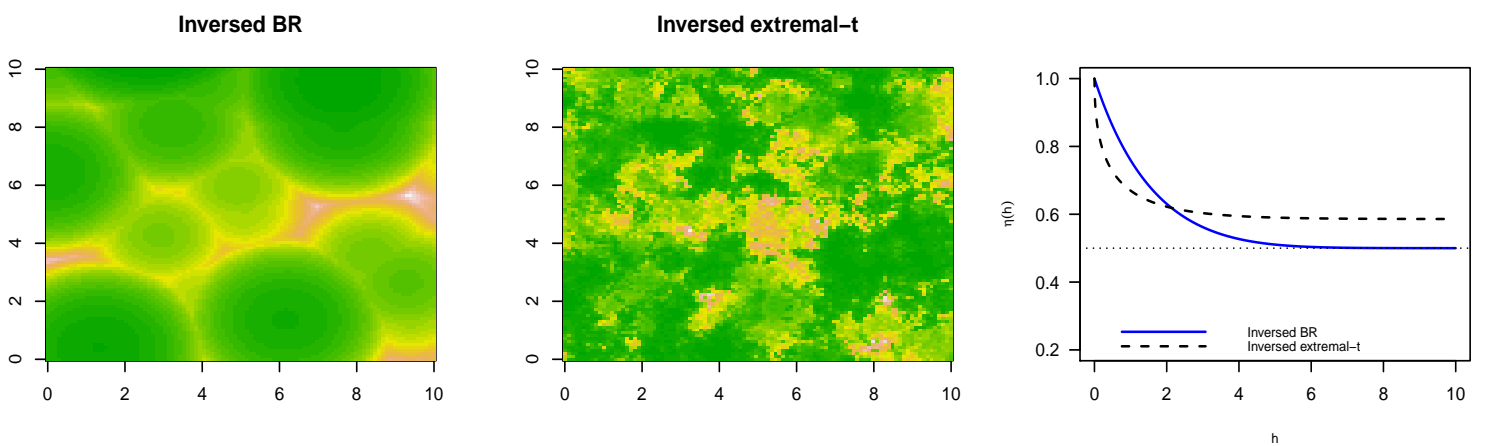

Figure 1. Simulations of two inverted max-stable processes (2.11) on the logarithm scale. Left panel: simulated image of AI process constructed by inverting an isotropic inverted Brown-Resnick process with variogram $2 \gamma(h)=(h / 1.5)^{2}$. Middle panel: simulated image of AI process constructed by inverting an isotropic extremal $-t$ process with $v=1$ degrees of freedom and exponential correlation function $\rho(h)=\exp (-h / 1.5)$. On the Right panel: associated functions $\eta(h)$.

Figure 2 displays five simulated images of the max-mixture model over the $[0,10]^{2}$ square according to different values of the mixing coefficient $a$. In order to show the role of the mixing 
coefficient, the values in the images are acquired by considering the simulation when $a=1$ (maxstable process) and $a=0$ (inverted max-stable process). It is noteworthy that the smoothness decreases as $a$ increases. Figure 16 (Appendix A) displays further examples of simulated images of max-mixture models.
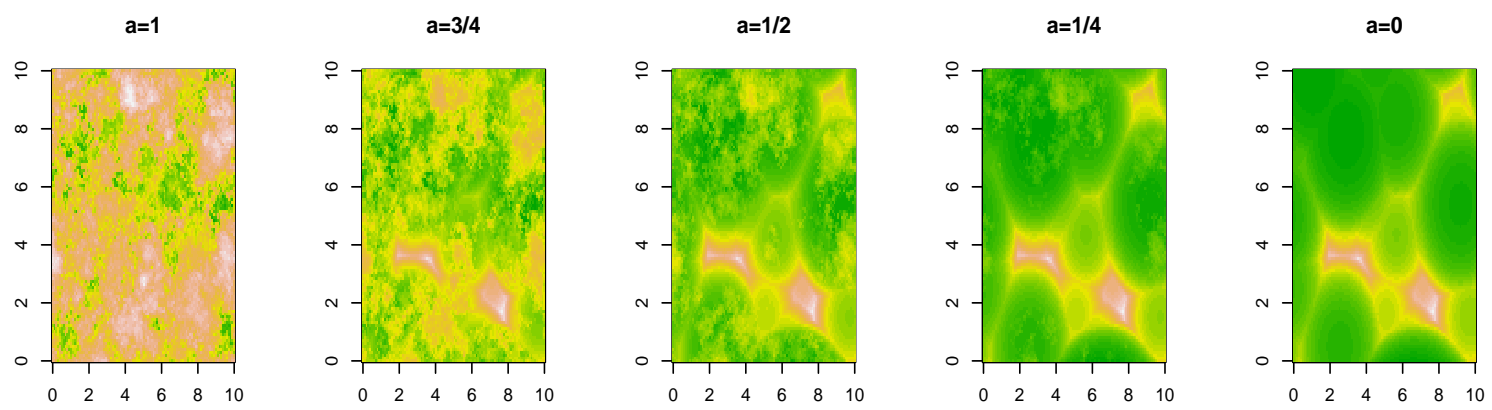

Figure 2. Simulations of the max-mixture model (2.12) on the logarithm scale according different values of mixing coefficient $a \in\{1,0.75,0.5,0.25,0\} . X$ is an isotropic extremal $-t$ process with $v=1$ degrees of freedom and exponential correlation function $\rho(h)=\exp (-h)$, and $Y$ is an isotropic inverted BrownResnick process with variogram $2 \gamma(h)=(h / 1.5)^{2}$.

Figure 3 illustrates how the spatial extremal dependencies vary regarding to $a$. Based on (2.13), the measure $\chi_{Z}$ associated to the process $Z$ can be computed for a distance $h$ as $\chi_{Z}(h)=$ $a \chi_{X}(h)$, see (Bacro et al., 2016). The hybrid model extends traditional dependence modeling within the $\mathrm{AD}$ class and is appropriate when $\mathrm{AD}$ is present at all distances because it permits to capture a second order in the dependence structure which is not possible with a max-stable model.
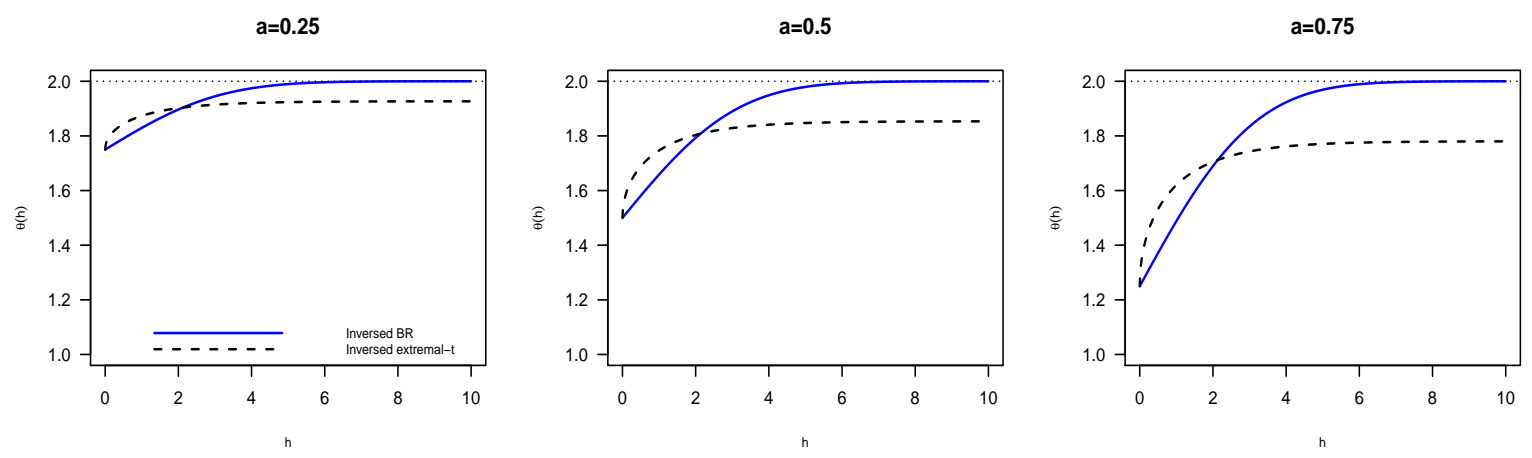

Figure 3. Associated $\theta($.$) functions for a max-mixture process Z($.$) based on two max-stable processes$ $X($.$) and X^{\prime}($.$) that belong to the same family with the same parameters used in Figure 1$ for $a \in$ $\{0.25,0.5,0.75\}$.

In (Bacro et al., 2016) daily rainfall data in the East of Australia are studied. Different models (MS, AI, and MM) are fitted to the data. It is showed that MM models has the merit to overcome the limits of MS models in which only $\mathrm{AD}$ or exact $\mathrm{AI}$ can be modeled. 


\section{3. $F^{\lambda}$-MADOGRAM FOR SPATIAL MAX-MiXTURE MODEL}

In the present paper we shall use the $F^{\lambda}$-madogram defined by Equation (2.10) for maxmixture models (2.12). We begin by calculating the expression of the $F^{\lambda}$-madogram for maxmixture models. Then, we shall develop a $F^{\lambda}$-madogram procedure to estimate $\theta_{X}, \theta_{Y}$ and chose $a$.

Proposition 3.1. Let $\{X(s), s \in \mathcal{S}\}$ be a simple max-stable process, with extremal coefficient function $\theta_{X}(h)$, and $Y(s)$ be an inverted max-stable process with coefficient of tail dependence function $\eta(h)=1 / \theta_{Y}(h)$. Let $a \in[0,1]$ and $Z=\max \{a X,(1-a) Y\}$. Then, the $F^{\lambda}$-madogram of the spatial max-mixture process $Z(s)$ is given by

$$
\begin{aligned}
& \nu_{F^{\lambda}}(h)=\frac{\lambda}{1+\lambda}-\frac{2 \lambda}{a\left(\theta_{X}(h)-1\right)+1+\lambda}+ \\
& \quad \frac{\lambda}{a \theta_{X}(h)+\lambda}-\frac{\lambda \theta_{Y}(h)}{(1-a) \theta_{Y}(h)+a \theta_{X}(h)+\lambda} \beta\left(\frac{a \theta_{X}(h)+\lambda}{1-a}, \theta_{Y}(h)\right)
\end{aligned}
$$

where $\beta(.,$.$) is the beta function.$

As a consequence of Proposition 3.1, we easily recover the expressions of $F^{\lambda}$-madograms for max-stable and inverted max-stable processes. The $F^{\lambda}$-madogram of a simple max stable process $X$ with extremal dependence coefficient $\theta_{X}$ is:

$$
\nu_{F^{\lambda}}(h)=\frac{\lambda}{\lambda+1} \frac{\theta_{X}(h)-1}{\lambda+\theta_{X}(h)}
$$

and we have $\nu_{F^{\lambda}}(h) \in\left[0, \frac{\lambda}{(1+\lambda)(2+\lambda)}\right]$. The $F^{\lambda}$-madogram of an inverted max-stable process $Y$ with extremal dependence coefficient $\theta_{Y}$ is:

$$
\nu_{F^{\lambda}}(h)=\frac{1}{1+\lambda}-\frac{\lambda \theta_{Y}(h)}{\lambda+\theta_{Y}(h)} \beta\left(\lambda, \theta_{Y}(h)\right)
$$

Proof. of Proposition 3.1 We use that for any $x, y \in \mathbb{R},|x-y|=2 \max \{x, y\}-(x+y)$ with $x=F^{\lambda}\{Z(s)\}$ and $y=F^{\lambda}\{Z(s+h)\}$. Moreover, recall that $\mathbb{E}\left[F^{\alpha}\{Z(s)\}\right]=1 /(1+\alpha)$. We have

$$
\begin{aligned}
\nu_{F^{\lambda}}(h) & =\mathbb{E}\left[\max \left\{F^{\lambda}\{Z(s)\}, F^{\lambda}\{Z(s+h)\}\right\}\right]-\frac{1}{2} \mathbb{E}\left[F^{\lambda}\{Z(s)\}+F^{\lambda}\{Z(s+h)\}\right] \\
& =\mathbb{E}\left[\max \left\{F^{\lambda}\{Z(s)\}, F^{\lambda}\{Z(s+h)\}\right\}\right]-\frac{1}{(1+\lambda)}
\end{aligned}
$$


Consider the random variable $W=\max \left\{F^{\lambda}\{Z(s)\}, F^{\lambda}\{Z(s+h)\}\right\}$. Then probability distribution function $G$ of $W$ satisfies

$$
\begin{aligned}
G(z) & =\mathbb{P}[W \leq z] \\
& =\mathbb{P}\left[\max \left\{F^{\lambda}\{Z(s)\}, F^{\lambda}\{Z(s+h)\}\right\} \leq z\right] \\
& =\mathbb{P}\left[Z(s) \leq F^{-\lambda}(z), Z(s+h) \leq F^{-\lambda}(z)\right] \\
& =\mathbb{P}\left[Z(s) \leq-\frac{\lambda}{\log (z)}, Z(s+h) \leq-\frac{\lambda}{\log (z)}\right] \\
& =\exp \left\{-V_{X}\left(-\frac{\lambda}{a \log (z)},-\frac{\lambda}{a \log (z)}\right)\right\} \cdot\left[z^{\frac{1-a}{\lambda}}+z^{\frac{1-a}{\lambda}}-1+\right. \\
& \left.\exp \left\{-V_{Y}\left(-\frac{1}{\log \left(1-z^{\frac{1-a}{\lambda}}\right)},-\frac{1}{\log \left(1-z^{\frac{1-a}{\lambda}}\right)}\right)\right\}\right]
\end{aligned}
$$

This rewrites:

$$
\begin{aligned}
G(z) & =z^{\frac{a \theta_{X}(h)}{\lambda}} \cdot\left[2 z^{\frac{(1-a)}{\lambda}}-1+\left(1-z^{\frac{(1-a)}{\lambda}}\right)^{\theta_{Y}(h)}\right] \\
& =2 z^{\frac{a\left(\theta_{X}(h)-1\right)+1}{\lambda}}-z^{\frac{a}{\lambda} \theta_{X}(h)}+z^{\frac{a}{\lambda} \theta_{X}(h)}\left(1-z^{\frac{(1-a)}{\lambda}}\right)^{\theta_{Y}(h)} .
\end{aligned}
$$

Thus, we are led to

$$
\begin{aligned}
\mathbb{E}[W] & =\int_{0}^{1} z d G \\
& =\left.z G\right|_{0} ^{1}-\int_{0}^{1} G d z \\
& =1-\left[\frac{2 \lambda}{a\left(\theta_{X}(h)-1\right)+1+\lambda}-\frac{\lambda}{a \theta_{X}(h)+\lambda}+I\right]
\end{aligned}
$$

and

$$
\begin{aligned}
I:=\int_{0}^{1} z^{\frac{a}{\lambda} \theta_{X}(h)}\left(1-z^{\frac{(1-a)}{\lambda}}\right)^{\theta_{Y}(h)} d z & =\frac{\lambda}{(1-a)} \beta\left(\frac{a \theta_{X}(h)+\lambda}{1-a}, \theta_{Y}(h)+1\right) \\
& =\frac{\lambda \theta_{Y}(h)}{(1-a) \theta_{Y}(h)+a \theta_{X}(h)+\lambda} \beta\left(\frac{a \theta_{X}(h)+\lambda}{1-a}, \theta_{Y}(h)\right)
\end{aligned}
$$

This proves Equation (3.1).

Figure 4 displays the behavior of the $F^{\lambda}$-madogram for three different max-mixture models with respect to the distance $h$ for different $\lambda \in\{0.5,1,1.5,3\} . \lambda=1.5$ corresponds to the 
largest values of the $F^{\lambda}$ madogram. Figure 17 (Appendix A) shows the curves of theortical $F^{\lambda}$-madogram for several other max-mixture processes.
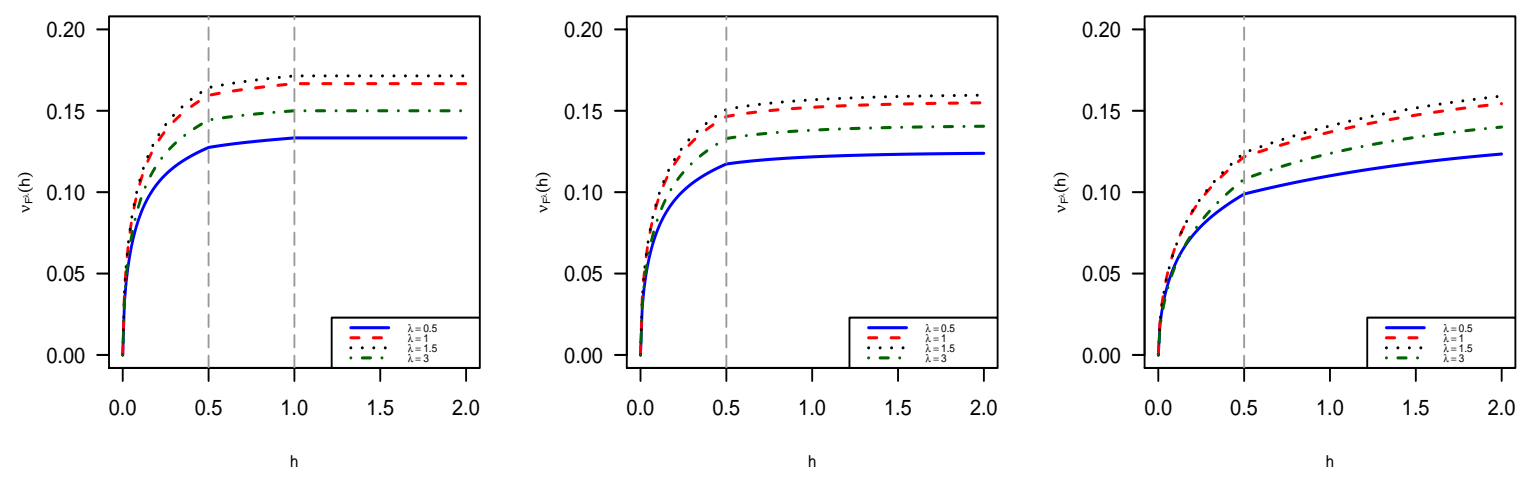

Figure 4. Theoretical $F^{\lambda}$-madogram functions 3.1 for $\lambda=0.5,1,1.5,3$. Left panel: Max-mixture model in which $X$ is a TEG process with $\mathcal{A}_{X}$ a disk of fixed radius $r_{X}=0.25$ and $\rho_{X}(h)=\exp (-h / 0.2)$. The asymptotic independent process $Y$ is an inverse TEG process with $\mathcal{A}_{Y}$ a disk of fixed radius $r_{Y}=0.5$ and $\rho_{Y}(h)=\exp (-h / 0.4)$. Middle panel: Max-mixture model in which $X$ is a TEG process as before. The asymptotic independent process $Y$ is an inverse extremal $-t$ process with $v=1$ degrees of freedom and $\rho_{Y}(h)=\exp (-h / 0.6)$. Right panel: Max-mixture model in which $X$ is a TEG process as before. $Y$ is an inverted Brown-Resnick process with semivariogram $\gamma(h)=h^{2}$. The mixing coefficient is setted to $a=0.5$ in the three models. The grey vertical lines represent the diameters of the disks for TEG processes.

We are now in position to describe a choice scheme for the mixing parameter $a$ of a maxmixture process $Z$ in (2.12). From Equation (3.1), we may write the $F^{\lambda}$-madogram as a function of $a, \lambda, \theta_{X}$ and $\theta_{Y}$, that is $\nu_{F^{\lambda}}(h)=\Phi\left(a, \lambda, \theta_{X}(h), \theta_{Y}(h)\right)$. The idea of our choice procedure is that $\theta_{X}$ and $\theta_{Y}$ may be estimated by $\widetilde{\theta}_{X}$ and $\widetilde{\theta}_{Y}$, minimizing the square difference between $\Phi\left(a, \lambda, \theta_{X}(h), \theta_{Y}(h)\right)$ and its empirical counterpart, then we can choose $a$ such that the empirical version of the $F^{\lambda^{\prime}}$-madogram is the closest to $\Phi\left(a, \lambda^{\prime}, \widetilde{\theta}_{X}(h), \widetilde{\theta}_{Y}(h)\right)$. This idea is close to the non parametric estimation of the parameters of MM processes that has been proposed in (Ahmed et al., 2017) as an alternative to maximum composite likelihood estimation.

Formally, we consider $Z_{i}, i=1, \ldots, N$ copies of $Z$,

$$
Q_{i}(h, \lambda)=\frac{1}{2}\left|F^{\lambda}\left(Z_{i}(s)\right)-F^{\lambda}\left(Z_{i}(s+h)\right)\right|,
$$

where $F$ denotes the unit Frchet distribution function. From the definition of the $F^{\lambda}$-madogram, we have $\mathbb{E}\left[Q_{i}(h, \lambda)\right]=\nu_{F^{\lambda}}(h)$. Denote by $\Lambda \subset[0, \infty)$ a finite set of some possible $\lambda$ choices, then for a given value of $a$, a semi-parametric nonlinear least squares minimization procedure for estimating the extremal coefficient $\boldsymbol{\theta}(h)=\left(\theta_{X}(h), \theta_{Y}(h)\right)^{t}$ is

$$
\widetilde{\boldsymbol{\theta}}_{\boldsymbol{N} \boldsymbol{L} \boldsymbol{S}}^{\boldsymbol{a}}(h)=\underset{\theta \in[1,2]^{2}}{\arg \min } N^{-1} \sum_{\lambda \in \Lambda} \sum_{i=1, \ldots, N}\left[Q_{i}(h, \lambda)-\Phi\left(a, \lambda, \theta_{X}(h), \theta_{Y}(h)\right)\right]^{2}
$$


Assume that the $Z_{i}$ 's are observed at locations $s_{1}, \ldots, s_{K}$ and let $h$ be the pairwise distances between the $s_{j}$ 's. We shall denote by $\widehat{\nu}_{F^{\lambda}}(h)$ the empirical version of $\nu_{F^{\lambda}}(h)$, that is for $\| s_{\ell}-$ $s_{p} \|=h$,

$$
\widehat{\nu}_{F^{\lambda}}(h)=\frac{1}{2 N} \sum_{i=1}^{N}\left|F^{\lambda}\left\{Z_{i}\left(s_{\ell}\right)\right\}-F^{\lambda}\left\{Z_{i}\left(s_{p}\right)\right\}\right| .
$$

For $a$ fixed, let $\widetilde{\boldsymbol{\theta}}_{\boldsymbol{N} \boldsymbol{L} \boldsymbol{S}}^{a}(h)=\left(\widetilde{\theta}_{X}^{a}(h), \widetilde{\theta}_{Y}^{a}(h)\right)^{t}$ be estimated as above with some chosen distinct values $\lambda \in \Lambda$. Let $\lambda^{\prime} \notin \Lambda$ and denote by $\mathcal{H} \subset[0, \infty)$ a finite set of spatial lags $h$. Moreover, let $\widetilde{\nu}_{F^{\lambda}}$ be the estimation of $\nu_{F^{\lambda}}$ where $\theta_{X}$ and $\theta_{Y}$ are replaced in (3.1) by $\widetilde{\theta}_{X}^{a}$ and $\widetilde{\theta}_{Y}^{a}$. We define:

$$
\operatorname{DC}(a)=\sum_{h \in \mathcal{H}} \omega(h)\left[\frac{\widehat{\nu}_{F^{\lambda^{\prime}}}(h)}{\widetilde{\nu}_{F^{\lambda^{\prime}}}(h)}-1\right]^{2}
$$

where the $\omega(h)$ 's are nonnegative weights which can be used for example to reduce the number of pairs included in the estimation. A simple choice for these weights is $\omega(h)=\mathbb{1}_{\{h \leq r\}}$ where the $r$ value can be chosen as the $q$-quantile of the distributions of the distances $h$ between pairs of sites, $q \in(0,1)$. Finally, we select $a$ that gives the lower value of (DC).

Of course, when dealing with real data, the marginal laws are usually not unit Frchet and thus have to be changed to unit Frchet. In that case, the empirical distribution function $\widehat{F}$ is used instead of $F$ in the definitions of $Q_{i}(h, \lambda)$ and $\widehat{\nu}_{F}^{\lambda}$ :

$$
\begin{aligned}
\widehat{Q_{i}}(h, \lambda) & =\frac{1}{2}\left|\widehat{F}^{\lambda}\left(Z_{i}(s)\right)-\widehat{F}^{\lambda}\left(Z_{i}(s+h)\right)\right|, \text { and } \\
\widehat{\widehat{\nu}}_{F^{\lambda}}(h) & =\frac{1}{2 N} \sum_{i=1}^{N}\left|\widehat{F}^{\lambda}\left\{Z_{i}\left(s_{\ell}\right)\right\}-\widehat{F}^{\lambda}\left\{Z_{i}\left(s_{p}\right)\right\}\right| .
\end{aligned}
$$

We shall denote $\widehat{\theta}_{X}^{a}, \widehat{\theta}_{Y}^{a}, \widehat{a}$ the estimations of $\theta_{X}, \theta_{Y}$ and $a$ obtained when $\widehat{Q}_{i}(h, \lambda)$ and $\widehat{\widehat{\nu}}_{F^{\lambda}}$ are used.

In order to get the consistency of our estimations, we need the two following assumptions:

- $I_{1}$ : for any $a \in[0,1],\left\{\lambda_{1}, \lambda_{2}\right\} \in \Lambda$ with $\lambda_{1} \neq \lambda_{2}$, the mapping

$$
\begin{aligned}
{[1,2]^{2} } & \longrightarrow \mathbb{R}^{2} \\
(x, y) & \mapsto\left(\Phi\left(a, \lambda_{1}, x, y\right), \Phi\left(a, \lambda_{2}, x, y\right)\right)
\end{aligned}
$$

is injective.

- $I_{2}$ : let $\theta_{X}$, (resp. $\left.\theta_{Y}\right), \theta_{X}^{\prime}$, (resp. $\theta_{Y}^{\prime}$ ) be extremal coefficients of max-stable (resp. inverse max-stable) processes. Let $\lambda$ be fixed, if for all $h, \Phi\left(a, \lambda, \theta_{X}(h), \theta_{Y}(h)\right)=$ $\Phi\left(a^{\prime}, \lambda, \theta_{X}^{\prime}(h), \theta_{Y}^{\prime}(h)\right)$ then $a=a^{\prime}$. 
Remark 3.2. The hypothesis $I_{1}$ and $I_{2}$ are identifiability hypothesis. Numerical tests on several models seem to indicate that they are satisfied for various max-mixtures models but we did not succeed to prove it.

Theorem 3.3. Assume that $\left(Z_{i}\left(s_{j}\right)\right)_{i=1, \ldots, N}$ are i.i.d copies of $Z\left(s_{j}\right), j=1, \ldots, K$ where $Z$ is a max-mixture spatial process with mixing coefficient $a_{0} \in[0,1]$. Assume that the injectivity conditions $I_{1}$ and $I_{2}$ are verified. Then the estimations of a by $\widetilde{a}$ and $\widehat{a}$ are consistent in the sense that

$$
\widetilde{a} \longrightarrow a_{0} \text { in probability as } N \rightarrow \infty \text { and } \widehat{a} \longrightarrow a_{0} \text { in probability as } N \rightarrow \infty \text {. }
$$

Proof. We shall give the proof for $\widehat{a}$, the proof for $\widetilde{a}$ is simpler and can be done along the same lines. We first begin by proving the consistency of $\widehat{\theta}_{X}^{a_{0}}$ and $\widehat{\theta}_{Y}^{a_{0}}$. Consider $\left\{\lambda_{1}, \lambda_{1}^{\prime}\right\} \in \Lambda$, with $\lambda_{1} \neq \lambda_{1}^{\prime}$. Write

$$
\begin{gathered}
\varepsilon_{h, i}^{1}=\widehat{Q}_{i}\left(h, \lambda_{1}\right)-\Phi\left(a_{0}, \lambda_{1}, \theta_{X}(h), \theta_{Y}(h)\right) \text { and } \\
\varepsilon_{h, i}^{2}=\widehat{Q}_{i}\left(h, \lambda_{1}^{\prime}\right)-\Phi\left(a_{0}, \lambda_{1}^{\prime}, \theta_{X}(h), \theta_{Y}(h)\right) .
\end{gathered}
$$

Using the convergence results from (Naveau et al., 2009) (Proposition 3), we have that

$$
\begin{gathered}
\frac{1}{N} \sum_{i=1}^{N} \varepsilon_{h, i}^{k} \rightarrow 0 \text { in probability, } k=1,2 \text { and } \\
\exists \sigma_{k} \geq 0 \text { such that } \frac{1}{N} \sum_{i=1}^{N}\left(\varepsilon_{h, i}^{k}\right)^{2} \rightarrow \sigma_{k}^{2} \text {, in probability, } k=1,2 .
\end{gathered}
$$

From this remark, following the lines of proof of Theorem II.5.1 in (Antoniadis et al., 1992), see also the proof of Theorem 4.1 in (Ahmed et al., 2017), we conclude, using the injectivity hypothesis $I_{1}$ that for any $h, \widehat{\theta}_{X}^{a_{0}}(h) \longrightarrow \theta_{X}(h)$ in probability and $\widehat{\theta}_{Y}^{a_{0}}(h) \longrightarrow \theta_{Y}(h)$ in probability. Now, consider $\left(a^{*}, \theta_{X}^{*}(h), \theta_{Y}^{*}(h)\right)$, a limit point of $\left(\widehat{a}, \widehat{\theta}_{X}^{\widehat{a}}(h), \widehat{\theta}_{Y}^{\widehat{a}}(h)\right)$. Since $\widehat{a}$ reaches the minimum of $D C$, we have $D C(\widehat{a}) \leq D C\left(a_{0}\right)$. The convergence of $\widehat{\theta}_{X}^{a_{0}}, \widehat{\theta}_{Y}^{a_{0}}$ and $\widehat{\widehat{\nu}}_{F^{\lambda^{\prime}}}$ from (Naveau et al., 2009) (Proposition 3) implies that $\lim _{N \rightarrow \infty} D C\left(a_{0}\right)=0$ so that $D C\left(a^{*}\right)=0$ which leads to $\Phi\left(a^{*}, \lambda^{\prime}, \theta_{X}^{*}(h), \theta_{Y}^{*}(h)\right)=\Phi\left(a_{0}, \lambda_{2}, \theta_{X}(h), \theta_{Y}(h)\right)$ with $w(h) \neq 0$ and thus $a^{*}=a_{0}$ by the injectivity condition $I_{2}$.

Remark 3.4. As seen by our selection scheme above, we have to determine the choices of $\lambda \in \Lambda$ and $\lambda^{\prime} \notin \Lambda$. After a lot of trials and as a compromise between accuracy and computation time we found that good results can be acheived with $\Lambda=\{1,3\}$ and $\lambda^{\prime}=1.5$ as shown by the simulation study, Section 4. 


\section{Simulation Study}

This section is devoted to a simulation study, in order to benchmark our estimation procedure. We shall consider two different max-mixture models.

$\mathbf{M}_{1}$ is a max-mixture model in which $X$ is a TEG process with $\mathcal{A}_{X}$ a disk of fixed radius $r_{X}$. The AI process $Y$ is an inverse TEG process with $\mathcal{A}_{Y}$ a disk of fixed radius $r_{Y}$. For simplicity, we choose stationary and isotropic exponential correlation functions, with range parameters $\phi_{X}, \phi_{Y}>0$ respectively. The model parameters vector is $\boldsymbol{\psi}=\left(r_{X}, \phi_{X}, a, r_{Y}, \phi_{Y}\right)^{t}$.

$\mathbf{M}_{\mathbf{2}}$ is a max-mixture model where $X$ is an isotropic Brown-Resnick process with variogram $2 \gamma(h)=\left(h / \phi_{X}\right)^{\tau}$ and $Y$ is an isotropic inverted extremal $-t$ process with $v$ degrees of freedom and exponential correlation function $\rho_{Y}(h)=\exp \left(-h / \phi_{Y}\right), \phi_{Y}>0$. The model parameters vector is $\boldsymbol{\psi}=\left(\phi_{X}, \tau, a, v, \phi_{Y}\right)^{t}$.

We summarize our simulation study procedure in the following steps.

Step 1. For each experiment, we consider a moderately sized data from the two max-mixture models described above with a true mixing coefficient $a_{0}, K=50$ sites randomly and uniformly distributed in the square $[0, L]^{2}, L \in \mathbb{N}$ and $N=2000$ independent replications at each site. Max-stable processes were simulated using SpatialExtremes package in $R$ (Ribatet et al., 2011) except for the TEG processes which have been simulated as in (Davison and Gholamrezaee, 2012). Each experiment was repeated $M=100$ times.

Step 2. For each data set in Step 1, we estimated the extremal dependence functions $\theta_{X}(h)$ and $\theta_{Y}(h)$ using the nonlinear least squares estimation criterion as described in (3.4), using $\widehat{Q}_{i}$. This step was performed with a set of different mixing coefficients including the true one $a_{0}$.

Step 3. We calculate our decision criterion $(\mathrm{DC}(a))$ with the estimated $\widehat{\theta}_{X}^{a}(h)$ and $\widehat{\theta}_{Y}^{a}(h)$ functions from Step 2. It is expected that this criterion will lead to the minimum values when the true parameter $a$ is used to estimate $\theta_{X}(h)$ and $\theta_{Y}(h)$ in Step 2. Since choosing a wrong mixing coefficient $a$ should lead to a bad estimation of $\theta_{X}(h)$ and $\theta_{Y}(h)$. Equal weights $\omega($. 
are used.

To assess the performance of the nonlinear least squares estimator $\widehat{\boldsymbol{\theta}}_{N L S}^{a}(h)$, we simulate data from max-mixture models as mentioned in Step 1. The performances of the estimators are given by the relative mean square error $\mathrm{MSE}_{\mathrm{rel}}$, for the $\frac{K(K-1)}{2}$ pairwise distances $h_{j}$ which are the distances between sites pairs $\left(s_{\ell}, s_{k}\right)$ : see (Bel et al., 2008) p. 171 for a similar definition in the multivariate context

$$
\operatorname{MSE}_{\mathrm{rel}}\left(h_{j}\right)=M^{-1} \sum_{i=1}^{M} \frac{\left(\widehat{\theta}^{a}\left(h_{j}\right)-\theta\left(h_{j}\right)\right)^{2}}{\theta\left(h_{j}\right)}, \quad j=1, \ldots, \frac{K(K-1)}{2} .
$$

Figures 7 - 10 display the estimation performances with respect to distance $h$ using the mixing coefficients $a=0.75,0.5,0.25$. For other max-mixture models we have similar results which are presented in the Supplementary Material (see Appendix B). Generally, our estimation procedure appears to work well for all considered max-mixture models. Moreover, the estimation of $\theta_{X}(h)$ becomes more precise as the mixing coefficient value increases, and vice versa for $\theta_{Y}(h)$.
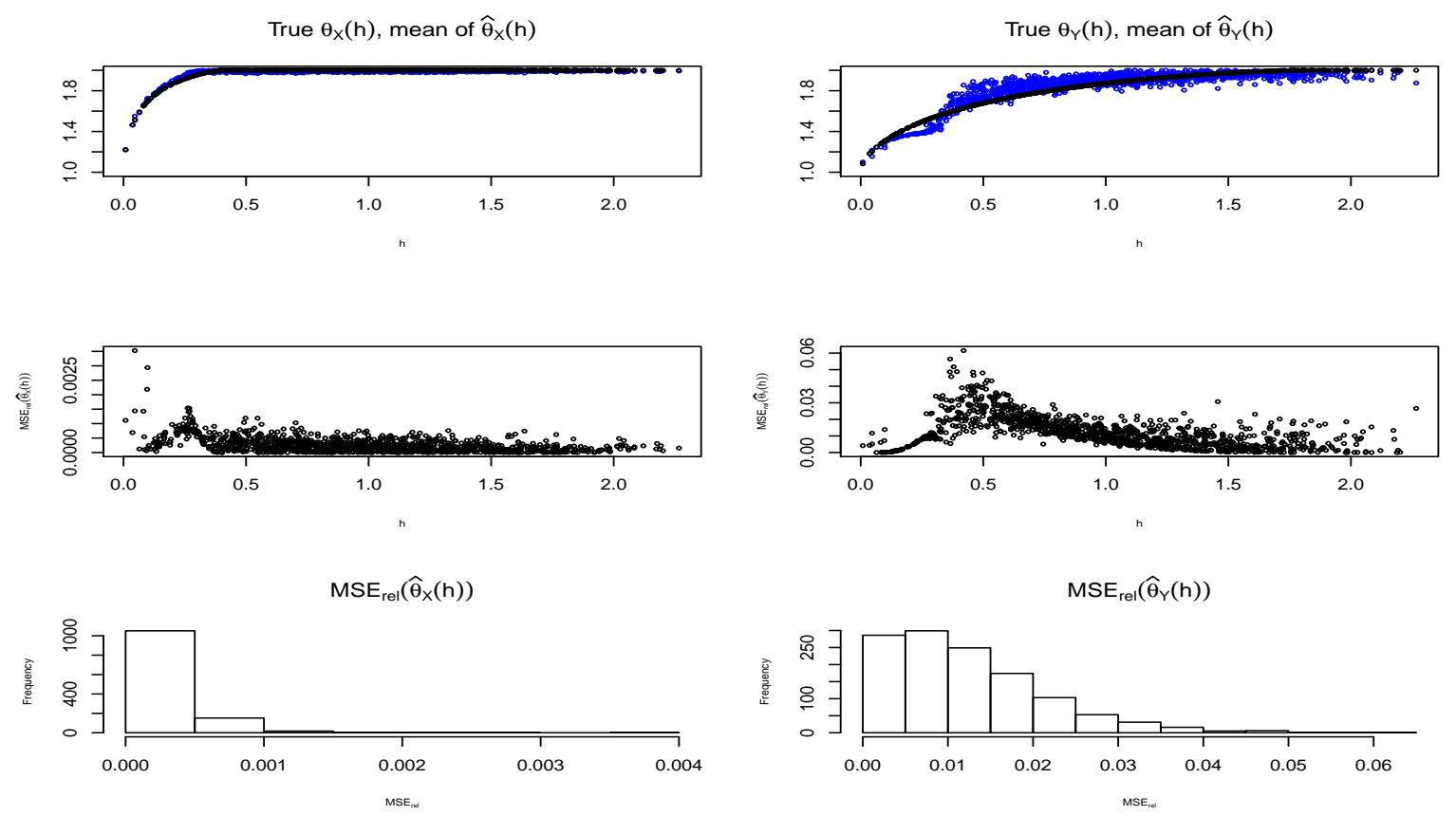

Figure 5. Estimation performance of $\hat{\boldsymbol{\theta}}_{N L S}(h)$ estimates. Data simulated from max-mixture model $\mathbf{M}_{\mathbf{1}}$ with parameter $\boldsymbol{\psi}=(0.2,0.1, \mathbf{0 . 7 5}, 0.9,0.7)^{t}$ in the square $[0,2]^{2}$ as described in Step 1 . The top row compares the true extermal coefficient functions (black points) and the NLS mean estimates (blue points) against the distance $h$. The middle row displays the MSE $\mathrm{rel}_{\text {of }}$ the NLS estimates against the distance $h$. While the bottom row displays the histograms of MSE $\mathrm{rel}_{\text {rel }}$ of the NLS estimates. 
True $\theta_{X}(h)$, mean of $\widehat{\theta}_{X}(h)$
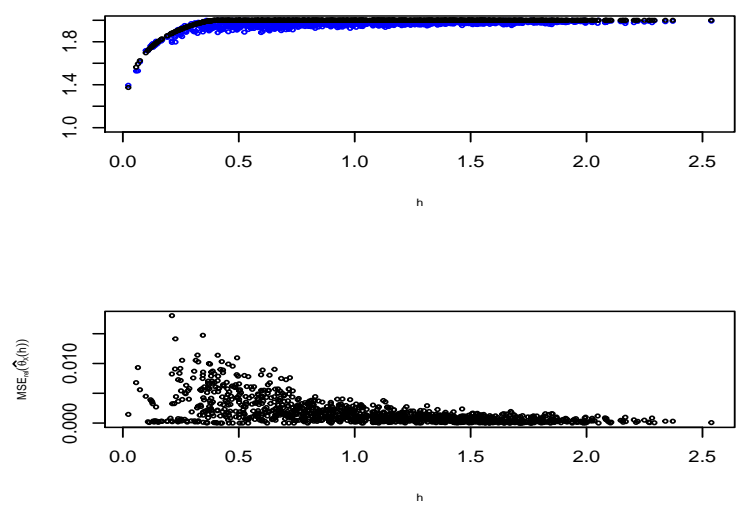

$\mathrm{MSE}_{\mathrm{rel}}\left(\widehat{\theta}_{\mathrm{X}}(\mathrm{h})\right)$

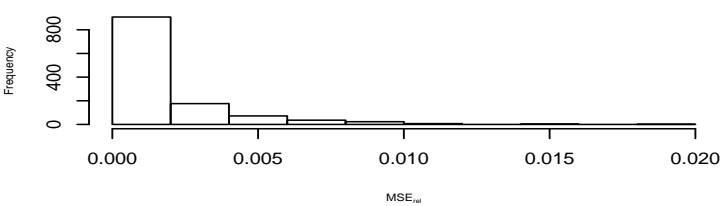

True $\theta_{Y}(h)$, mean of $\widehat{\theta}_{Y}(h)$
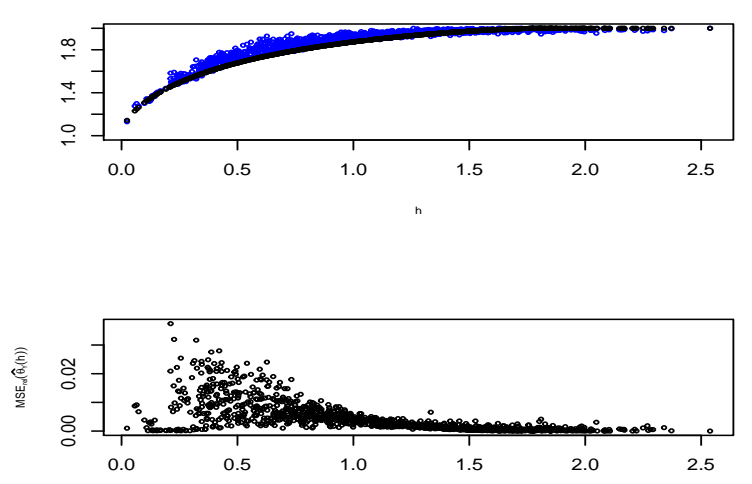

$\operatorname{MSE}_{\mathrm{rel}}\left(\widehat{\theta}_{\mathrm{Y}}(\mathrm{h})\right)$

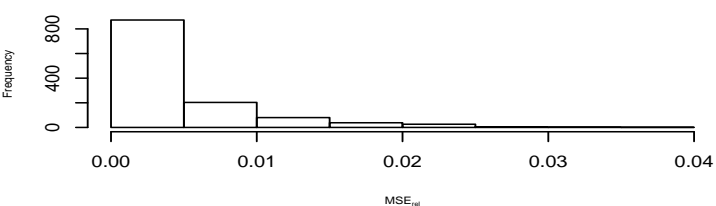

Figure 6. Estimation performance of $\hat{\boldsymbol{\theta}}_{N L S}(h)$ estimates. Data simulated from max-mixture model $\mathbf{M}_{\mathbf{1}}$ with parameter $\boldsymbol{\psi}=(0.2,0.1, \mathbf{0 . 5}, 0.9,0.7)^{t}$ in the square $[0,2]^{2}$ as described in Step 1 . The top row compares the true extermal coefficient functions (black points) and the NLS mean estimates (blue points) against the distance $h$. The middle row displays the MSE rel $_{\text {of }}$ the NLS estimates against the distance $h$. While the bottom row displays the histograms of MSE $\mathrm{M}_{\text {rel }}$ of the NLS estimates.

True $\theta_{x}(h)$, mean of $\widehat{\theta}_{x}(h)$
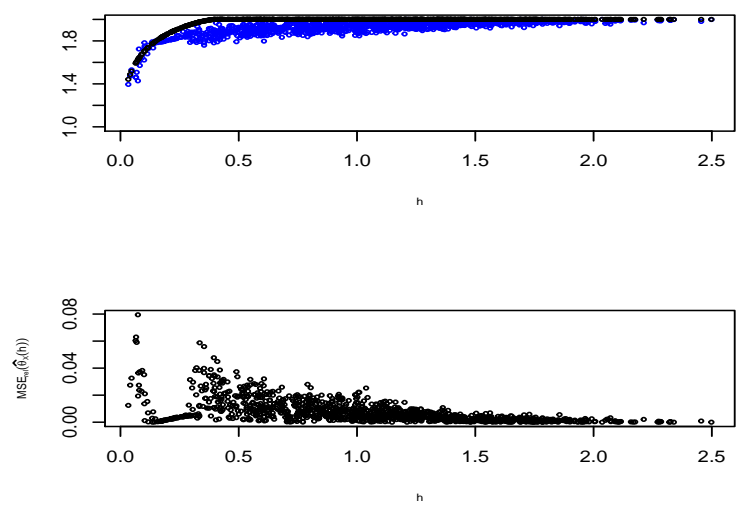

$\mathrm{MSE}_{\mathrm{rel}}\left(\widehat{\theta}_{\mathrm{X}}(\mathrm{h})\right)$

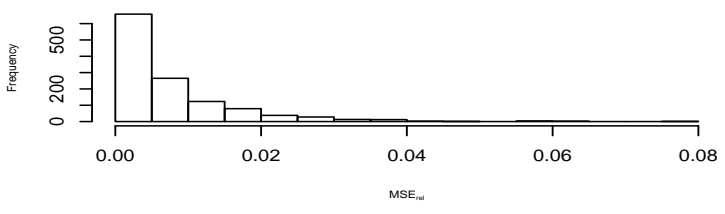

True $\theta_{Y}(\mathrm{~h})$, mean of $\widehat{\theta}_{Y}(\mathrm{~h})$
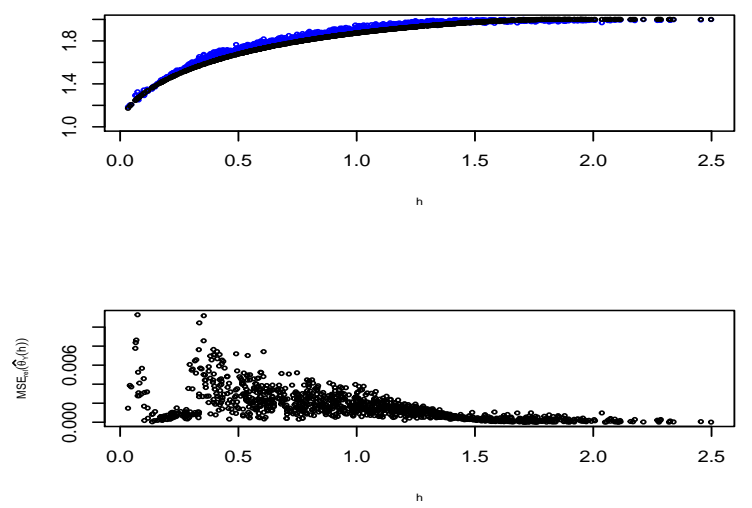

$\operatorname{MSE}_{\text {rel }}\left(\widehat{\theta}_{Y}(h)\right)$

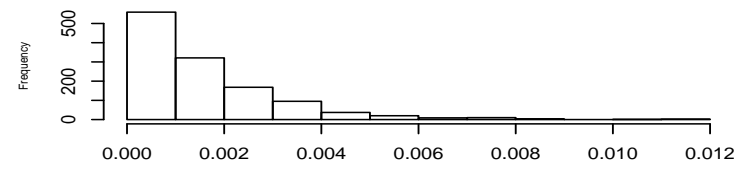

Figure 7. Estimation performance of $\hat{\boldsymbol{\theta}}_{N L S}(h)$ estimates. Data simulated from max-mixture model $\mathbf{M}_{1}$ with parameter $\boldsymbol{\psi}=(0.2,0.1, \mathbf{0 . 2 5}, 0.9,0.7)^{t}$ in the square $[0,2]^{2}$ as described in Step 1 . The top row compares the true extermal coefficient functions (black points) and the NLS mean estimates (blue points) against the distance $h$. The middle row displays the MSE $\mathrm{r}_{\mathrm{rel}}$ of the NLS estimates against the distance $h$. While the bottom row displays the histograms of MSE rel $_{\text {of }}$ the NLStimates. 
True $\theta_{X}(h)$, mean of $\widehat{\theta}_{X}(h)$
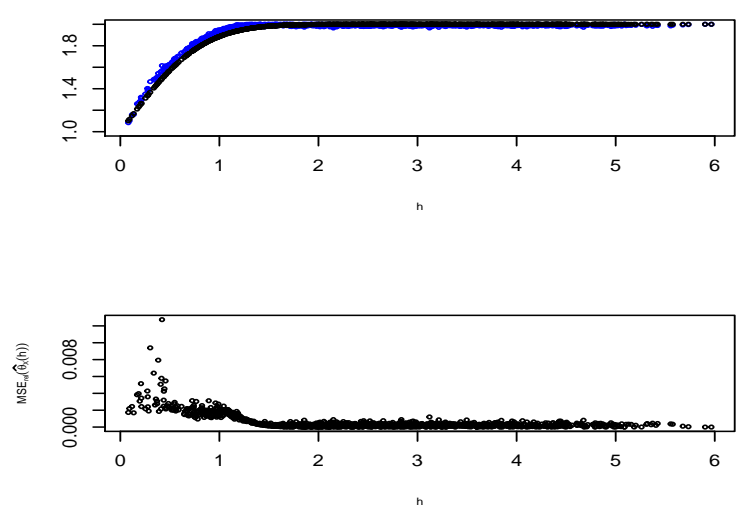

$\operatorname{MSE}_{\mathrm{rel}}\left(\widehat{\theta}_{\mathrm{X}}(\mathrm{h})\right)$

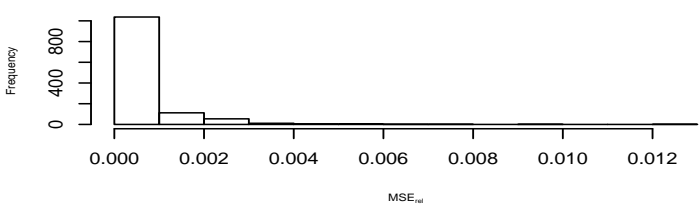

True $\theta_{\mathrm{Y}}(\mathrm{h})$, mean of $\widehat{\theta}_{\mathrm{Y}}(\mathrm{h})$
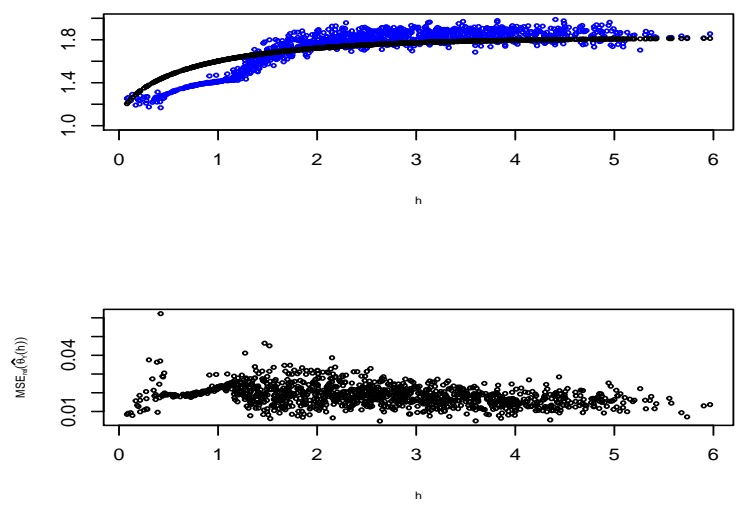

$\operatorname{MSE}_{\mathrm{rel}}\left(\widehat{\theta}_{\mathrm{Y}}(\mathrm{h})\right)$

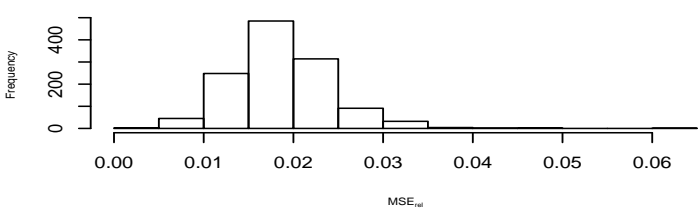

Figure 8. Estimation performance of $\hat{\boldsymbol{\theta}}_{N L S}(h)$ estimates. Data simulated from max-mixture model $\mathbf{M}_{\mathbf{2}}$ with parameter $\boldsymbol{\psi}=(0.1,2, \mathbf{0 . 7 5}, 2,1.5)^{t}$ in the square $[0,5]^{2}$ as described in Step 1. The top row compares the true extermal coefficient functions (black points) and the NLS mean estimates (blue points) against the distance $h$. The middle row displays the $\mathrm{MSE}_{\mathrm{rel}}$ of the NLS estimates against the distance $h$. While the bottom row displays the histograms of MSE $\mathrm{rel}_{\text {rel }}$ of the NLS estimates.

True $\theta_{x}(h)$, mean of $\widehat{\theta}_{\times}(h)$
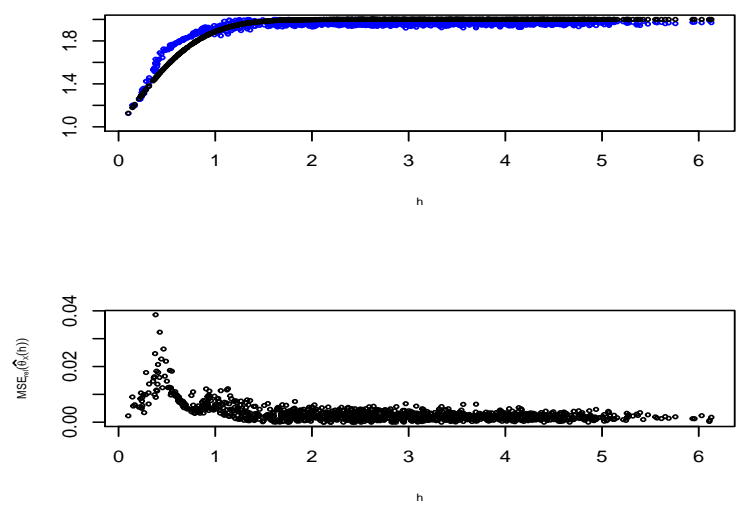

$\operatorname{MSE}_{\text {rel }}\left(\widehat{\theta}_{X}(h)\right)$

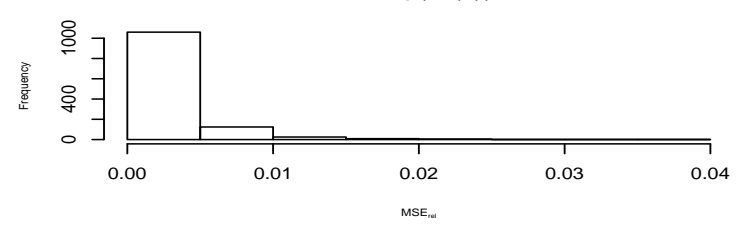

True $\theta_{\mathrm{Y}}(\mathrm{h})$, mean of $\widehat{\theta}_{\mathrm{Y}}(\mathrm{h})$
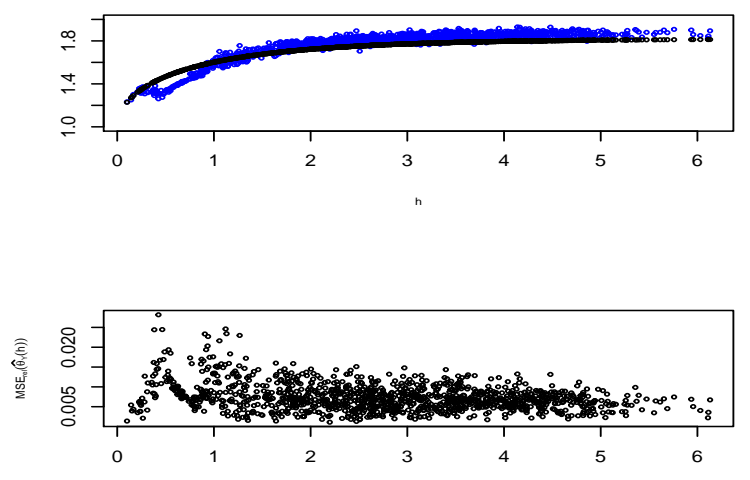

$\operatorname{MSE}_{\mathrm{rel}}\left(\widehat{\theta}_{\mathrm{Y}}(\mathrm{h})\right)$

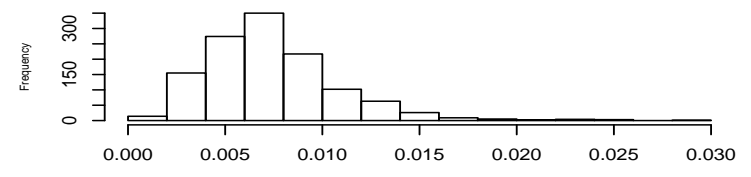

Figure 9. Estimation performance of $\hat{\boldsymbol{\theta}}_{N L S}(h)$ estimates. Data simulated from max-mixture model $\mathbf{M}_{2}$ with parameter $\boldsymbol{\psi}=(0.1,2, \mathbf{0 . 5}, 2,1.5)^{t}$ in the square $[0,5]^{2}$ as described in Step 1 . The top row compares the true extermal coefficient functions (black points) and the NLS mean estimates (blue points) against the distance $h$. The middle row displays the MSE $\mathrm{rel}_{\text {of }}$ the NLS estimates against the distance $h$. While the bottom row displays the histograms of MSE rel $_{\text {of }}$ the NLStimates. 
True $\theta_{\times}(h)$, mean of $\widehat{\theta}_{\times}(h)$
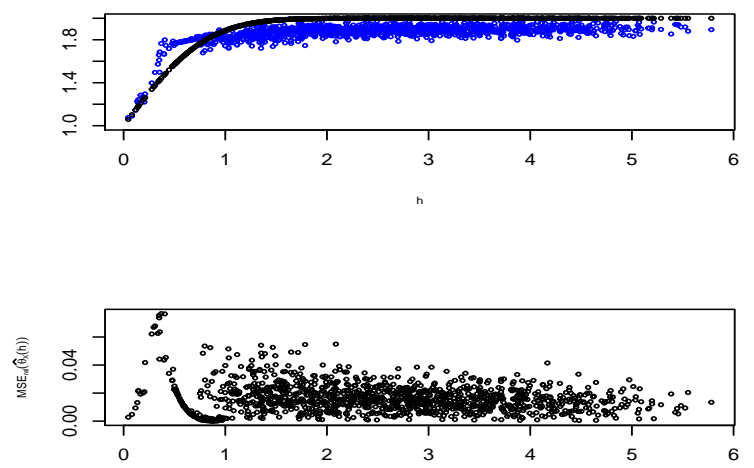

$\mathrm{MSE}_{\mathrm{rel}}\left(\widehat{\theta}_{\mathrm{X}}(\mathrm{h})\right)$

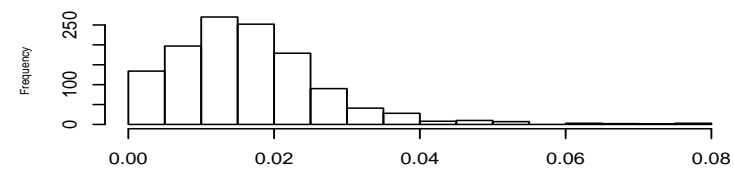

MSE
True $\theta_{Y}(h)$, mean of $\widehat{\theta}_{Y}(h)$
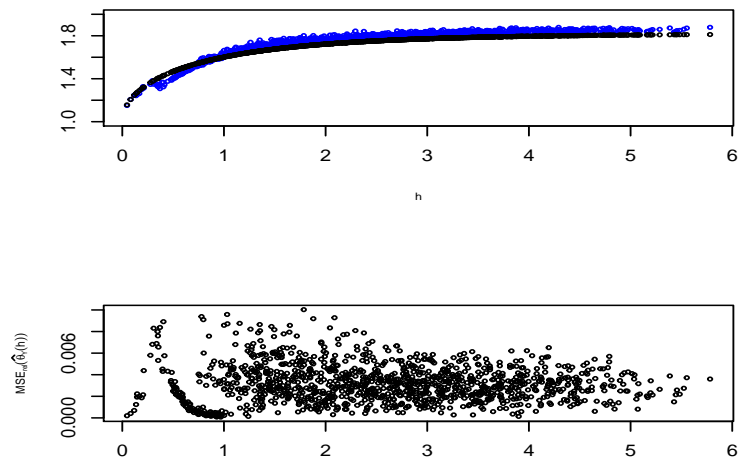

$\operatorname{MSE}_{\mathrm{rel}}\left(\widehat{\theta}_{\mathrm{Y}}(\mathrm{h})\right)$

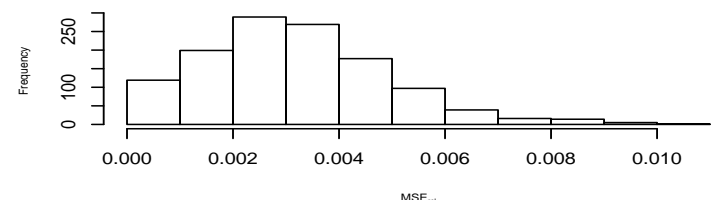

Figure 10. Estimation performance of $\hat{\boldsymbol{\theta}}_{N L S}(h)$ estimates. Data simulated from max-mixture model $\mathbf{M}_{\mathbf{2}}$ with parameter $\boldsymbol{\psi}=(0.1,2, \mathbf{0 . 2 5}, 2,1.5)^{t}$ in the square $[0,5]^{2}$ as described in Step 1 . The top row compares the true extermal coefficient functions (black points) and the NLS mean estimates (blue points) against the distance $h$. The middle row displays the MSE rel $_{\text {of }}$ the NLS estimates against the distance $h$. While the bottom row displays the histograms of $\mathrm{MSE}_{\mathrm{rel}}$ of the NLS estimates.

Now, we turn to our proposed model selection criterion (DC) for selecting the max-mixture models with the best mixing coefficient $a$ through a number of simulation studies using the two mentioned max-mixture models. The boxplots in Figure 11 display the values of the decision criterion (DC) against different mixing coefficients $a \in\{0,0.25,0.5,0.75,1\}$ for models $\mathbf{M}_{\mathbf{1}}$ and $\mathbf{M}_{\mathbf{2}}$. The lower values of DC are likely related with the true mixing coefficient $a_{0}$. Boxplots of the decision criterion (DC) for other examples of max-mixture models in which we have similar results available in the Supplementary Material (see Appendix B). 

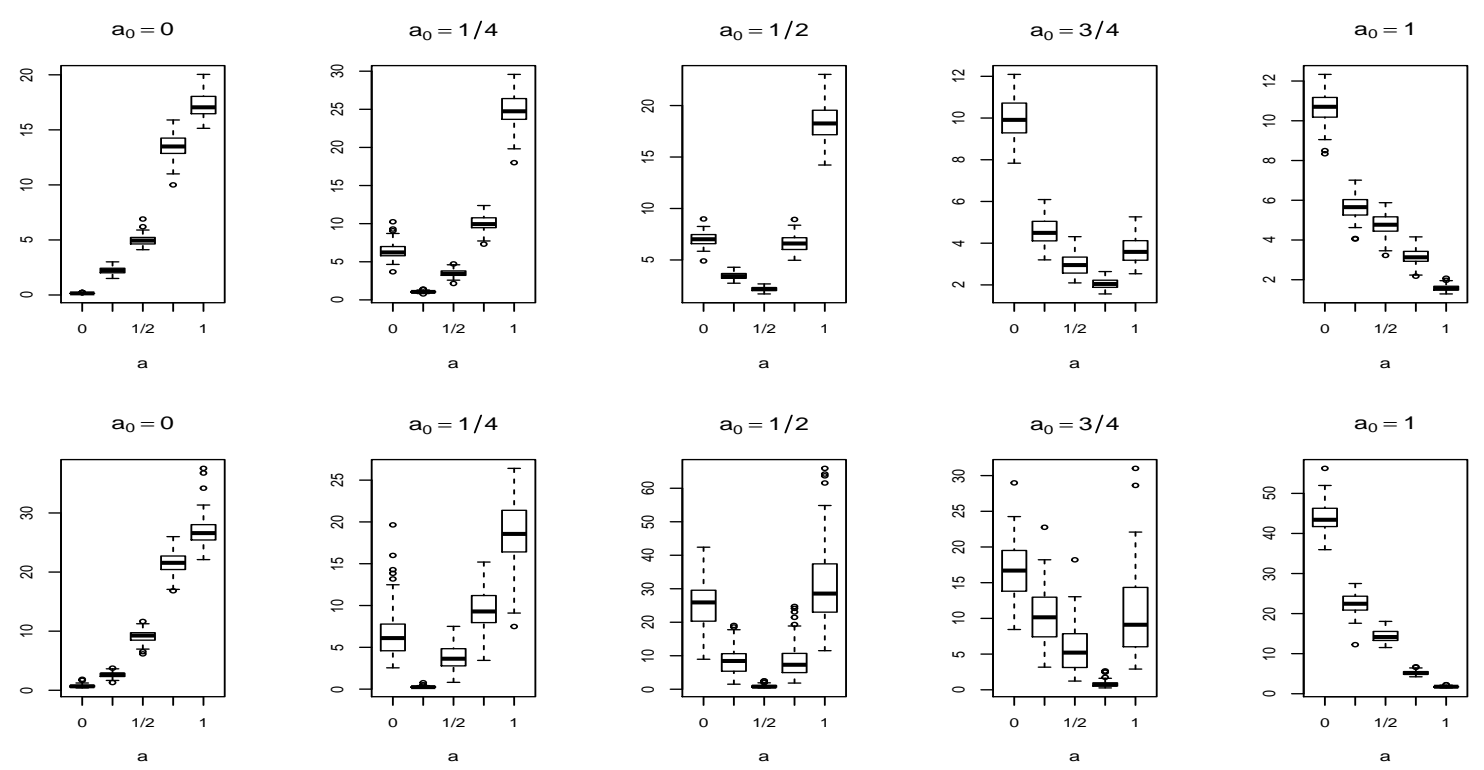

Figure 11. Top row: boxplots for decision criterion of 100 data replications of 2000 independent copies from $\mathbf{M}_{1}$ with parameters $\phi_{X}=0.1, r_{X}=0.2, \phi_{Y}=0.7$ and $r_{Y}=0.9$ over the square $[0,2]^{2}$. Bottom row: boxplots for decision criterion of 100 data replications of 2000 independent copies from $\mathbf{M}_{\mathbf{2}}$ with parameters $\phi_{X}=0.1, \tau=2, v=2$ and $\phi_{Y}=1.5$ over the square $[0,5]^{2}$.

\section{REAL DATA EXAMPLE}

The data analysed in this section are daily rainfall amounts in (millimetres) over the years 1972-2016 occurring during April-September at 38 sites in the East of Australia whose locations are shown in Figure 12. The altitude of the sites varying from 4 to 552 meters above mean sea level. The sites are separated by distances of approximately (34-1383) km. This data set is freely available on http://www.bom.gov.au/climate/data/index.shtml?bookmark=136. 


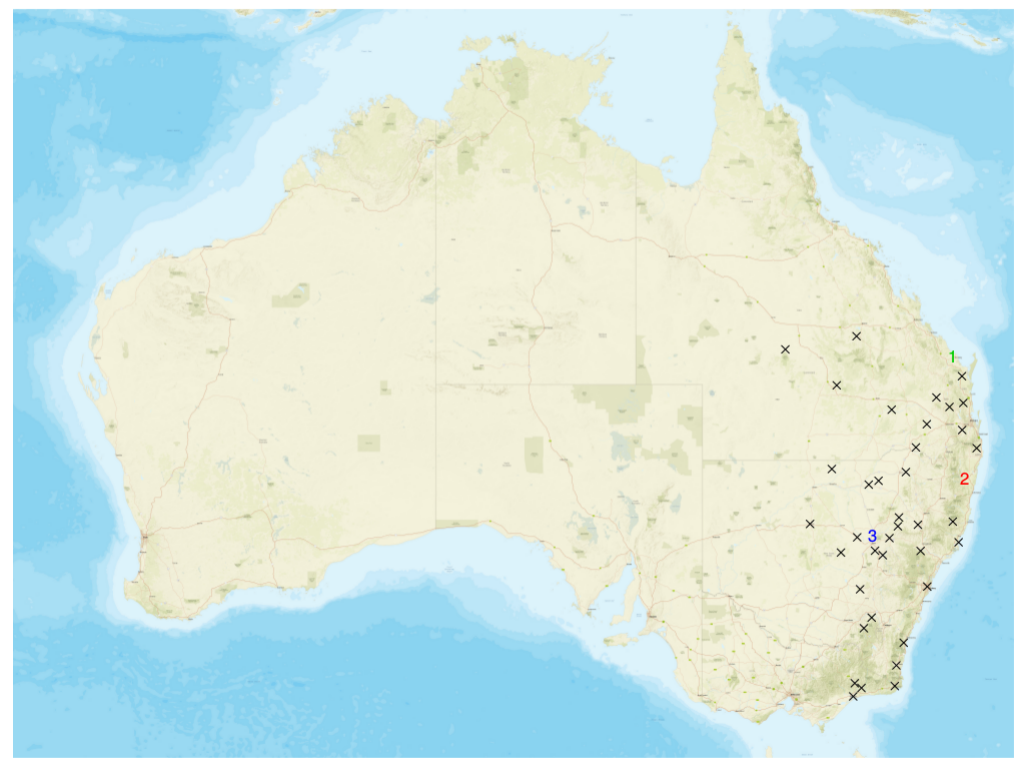

Figure 12. Geographical locations of 41 meteorological stations located in the East of Australia. Black crosses represent the 38 stations used for selecting the mixing coefficient $a$. The three colored numbers $\{1,2,3\}$ correspond to the unused stations on which we shall predict conditional probabilities in order to validate our procedure.

Following the approach of (Bacro et al., 2016) graphical assessments to explore possible anisotropy Figure 13 based on the empirical estimates of the functions $\chi(h, u)$ and $\bar{\chi}(h, u)$ in different directional sectors $(-\pi / 8, \pi / 8],(\pi / 8,3 \pi / 8],(3 \pi / 8,5 \pi / 8]$, and $(5 \pi / 8,7 \pi / 8]$, where 0 represents the northing direction. Based on these estimates there is no clear evidence of anisotropy.
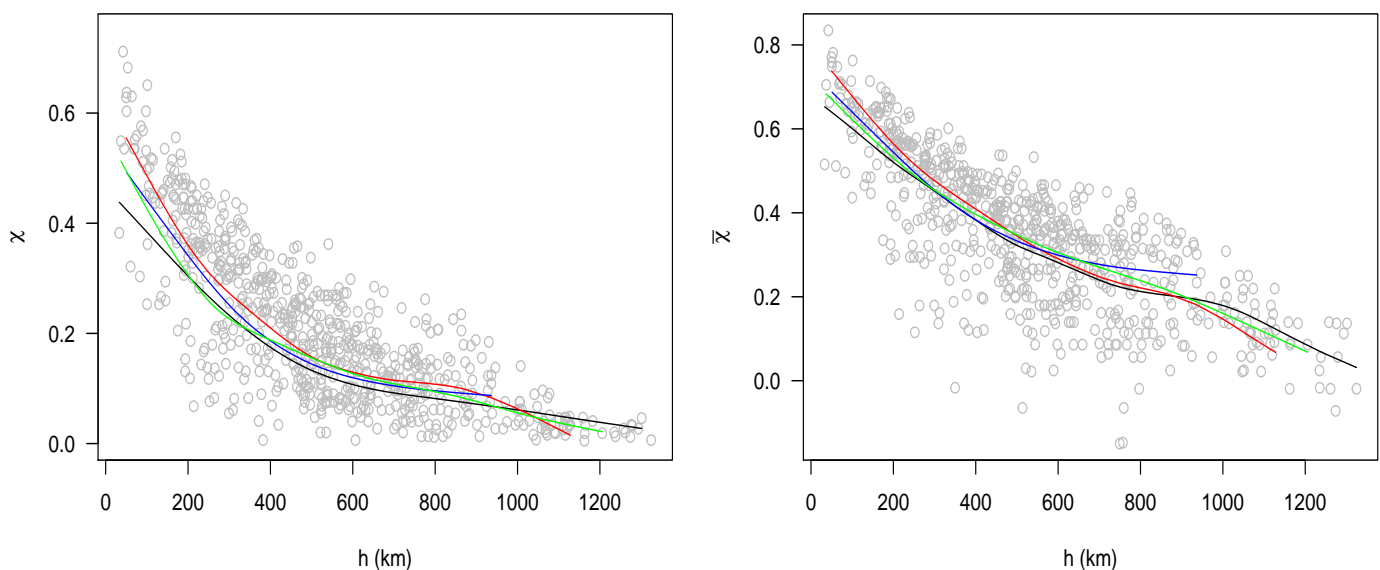

Figure 13. Pairwise empirical estimates of $\chi$ (left panel) and $\bar{\chi}$ (right panel) versus distance at threshold $u=0.970$. Grey points are empirical pairwise estimates for all data pairs. Colored lines are the loess smoothed values of the empirical estimates in different directional sectors: black line $(-\pi / 8, \pi / 8]$, red line $(\pi / 8,3 \pi / 8]$, blue line $(3 \pi / 8,5 \pi / 8]$, and green line $(5 \pi / 8,7 \pi / 8]$. 
We apply our methodology for the selection of the mixing coefficient for all $a \in(0,1)$ by steps 0.01. The $a \mapsto D C(a)$ function is plotted in Figure 14. The best-fitting max-mixture model as judged by our DC criterium has a mixing coefficient $a=0.34$.

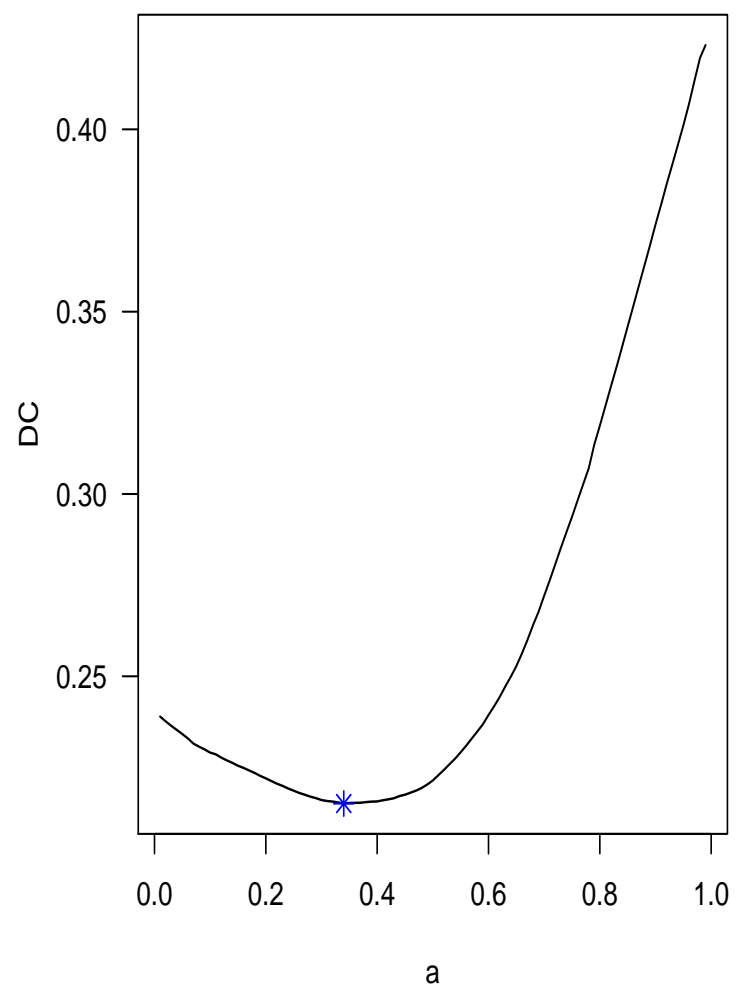

Figure 14. Decision criterion values for real data example on the interval $a \in(0,1)$ by steps 0.01 . The blue star corresponds to the minimum DC value which occurs at $a=0.34$.

In the literature, the widely used parametric inference procedure is based on composite likelihood methods. In particular, pairwise likelihood estimation has been found usefull to estimate parameters in a max-stable process. A description of this method can be found in (Padoan et al., 2010; Wadsworth and Tawn, 2012; Bacro et al., 2016) for spatial context.

Unfortunately, parameter estimation using composite likelihood suffers from some defects. First, it can be onerous, since the computation and subsquent optimization of the objective function is time-consuming. Second, the choice of good initial values for optimization of the composite likelihood is essential. Third, model dependency, a preliminary step to conduct a composite likelihood estimation is to specify the model that describes the dependence structure. So, this mission seems to be laborious, due to the large number of combinations that 
can be formed from the $\mathrm{AD}$ and $\mathrm{AI}$ processes stemming from max-stable processes, since the variety of dependence structures that can be assumed, i.e. changing the correlation coefficient function type in Schlather model (Schlather, 2002) leads to different dependence structures. So, an unacurate choice may lead to severe under/over estimations of probabilities associated to simultaneous extreme events.

In the sequel, we shall estimate the conditional probability of having daily rainfall that exceeds some threshold $z$ at an unused site denoted by $s_{0}^{*}$ given that this event has occurred at the nearest observed site which is denoted by $s_{0}$, i.e., $\mathbb{P}\left[Z\left(s_{0}^{*}\right)>z \mid Z\left(s_{0}\right)>z\right]$. We compare this estimation with that obtained by the best-fitting parametric model based on composite likelihood estimation.

For this purpose, we fitted the daily rainfall data based on censored pairwise likelihood approach used by (Wadsworth and Tawn, 2012; Bacro et al., 2016) where the threshold is taken corresponding to the 0.9 empirical quantile at each site. We fitted the generalized extreme value distribution $\operatorname{GEV}(\mu, \sigma, \xi)$ separately to each site and then data are transformed to unit Fréchet margins through the transformation $z \rightarrow \frac{-1}{\log (\widehat{G}(z))}$, where $\widehat{G}($.$) is the estimated GEV cumulative$ distribution function. The models are

$\mathbf{M}_{\mathbf{a}}$ : a MM model where $X$ is a TEG process with an exponential correlation function $\rho(h)=\exp \left(-\|h\| / \phi_{X}\right), \phi_{X}>0 . \mathcal{A}_{X}$ is a disk of fixed and unknown radius $r_{X}$, and $Y$ is an inverted TEG process with exponential correlation function $\rho(h)=\exp \left(-\|h\| / \phi_{Y}\right), \phi_{Y}>0$, and $\mathcal{A}_{Y}$ is a disk with fixed and unknown radius $r_{Y}$.

$\mathbf{M}_{\mathbf{b}}$ : a MM model where $X$ is a TEG process as in $\mathbf{M}_{\mathbf{a}} \cdot Y$ is an isotropic inverted Smith process where $\Sigma$ is a diagonal matrix $\left(\sigma_{12}=0\right)$ with $\sigma_{11}^{2}=\sigma_{22}^{2}=\phi_{Y}^{2}$, i.e., $\gamma(h)=\left(\|h\| / \phi_{Y}\right)$.

$\mathbf{M}_{\mathbf{c}}$ : a MS TEG process described as $X$ in $\mathbf{M}_{\mathbf{a}}$.

$\mathbf{M}_{\mathbf{d}}$ : a MS isotropic Smith process where $\Sigma$ is a diagonal matrix $\left(\sigma_{12}=0\right)$ with $\sigma_{11}^{2}=\sigma_{22}^{2}=$ $\phi_{X}^{2}$, i.e., $\gamma(h)=\left(\|h\| / \phi_{X}\right)$. 
$\mathbf{M}_{\mathbf{e}}$ : the inverted Smith process described as $Y$ in $\mathbf{M}_{\mathbf{b}}$.

The composite likelihood information criterion (CLIC) (Varin and Vidoni, 2005), defind as $\mathrm{CLIC}=-2\left[p \ell(\widehat{\vartheta})-\operatorname{tr}\left\{\mathcal{J}(\widehat{\vartheta}) \mathcal{H}^{-1}(\widehat{\vartheta})\right\}\right]$ is used to judge the best-fitting model. Here, the maximum pairwise likelihood estimator is denoted by $\widehat{\vartheta}$. Lower values of CLIC indicate a better fit. Our results are summarised in Table 1 . The best-fitting model for the data, as judged by CLIC, is the hybrid dependence model $\mathbf{M}_{\mathbf{b}}$.

Table 1. Parameter estimates of selected dependence models fitted to the daily rainfall data. The composite likelihood criterion (CLIC) and standard errors reported between parentheses. $(*)$ indicates to the lower CLIC.

\begin{tabular}{lllllll}
\hline Model & $\widehat{\phi}_{X}$ & $\widehat{r}_{X}$ & $\widehat{a}$ & $\widehat{\phi}_{Y}$ & $\widehat{r}_{Y}$ & CLIC \\
\hline $\mathbf{M}_{\mathbf{a}}$ & $254.66(179.69)$ & $683.32(257.18)$ & $0.59(0.21)$ & $1609.42(141.53)$ & $981.73(168.01)$ & 4515964 \\
$\mathbf{M}_{\mathbf{b}}$ & $93.16(48.02)$ & $166.92(80.86)$ & $0.27(0.14)$ & $971.65(243.29)$ & - & $4515911^{*}$ \\
$\mathbf{M}_{\mathbf{c}}$ & $188.49(53.27)$ & $691.12(215.53)$ & - & - & - & 4523182 \\
$\mathbf{M}_{\mathbf{d}}$ & $463.52(216.54)$ & - & - & - & - & 4523446 \\
$\mathbf{M}_{\mathbf{e}}$ & - & - & - & $628.38(86.54)$ & - & 4515981 \\
\hline
\end{tabular}

Now, we shall use our least square estimations of $a, \theta_{X}$ and $\theta_{Y}$ in order to estimate the conditional probabilities. The following lemma is easily deduced from (2.14); see the proof in Appendix C.

Lemma 5.1. Let $Z$ be a max-mixture process. Its bivariate tail distribution is given by

$$
\mathbb{P}\left[Z\left(s_{0}^{*}\right)>z \mid Z\left(s_{0}\right)>z\right]=\frac{1-2 e^{-\frac{1}{z}}+e^{-\frac{a \theta_{X}\left(h_{0}\right)}{z}}\left\{-1+2 e^{-\frac{1-a}{z}}+\left[1-e^{-\frac{1-a}{z}}\right]^{\theta_{Y}\left(h_{0}\right)}\right\}}{1-e^{-\frac{1}{z}}}
$$

where $h_{0}$ is the separation distance between $s_{0}^{*}$ and $s_{0}$. Equation (5.1) may be used to estimate $\mathbb{P}\left[Z\left(s_{0}^{*}\right)>z \mid Z\left(s_{0}\right)>z\right]$ using both parametric and nonparametric approaches. We consider $s_{0}^{*}$ as the three unused sites that have been marked by colored numbers $\{1,2,3\}$ on the map Figure 12 .

The threshold $z$ is taken corresponding to the $q$ - emperical quantile at the site $s_{0}, q \in(0,1)$. For estimating $\mathbb{P}\left[Z\left(s_{0}^{*}\right)>z \mid Z\left(s_{0}\right)>z\right]$ nonparametrically, we fitted the data again by NLS procedure with the best mixing coefficient $a=0.34$ and we obtained the estimators $\widehat{\theta}_{X}\left(h_{0}\right)$ and $\widehat{\theta}_{Y}\left(h_{0}\right)$ by averaging the values of $\widehat{\theta}_{X}(h)$ and $\widehat{\theta}_{Y}(h)$, where $h \in\left[h_{0}-10, h_{0}+10\right] \mathrm{km}$ by taking the advantage of stationarity and isotropy of our data and $h_{0} \in\{54.226,92.534,133.673\} \mathrm{km}$. While, 
for estimating $\mathbb{P}\left[Z\left(s_{0}^{*}\right)>z \mid Z\left(s_{0}\right)>z\right]$ parametrically, $\widehat{\theta}_{X}(h)$ and $\widehat{\theta}_{Y}(h)$ are obtained by substituting the separating distance $h_{0}$ and the estimated parameters from hybrid model $\mathbf{M}_{\mathbf{b}}$ Table 1 .

In order to compare the results obtained by the two approaches, we used the data at the unused three sites to compute the empirical version of conditional probabilities $\mathbb{P}\left[Z\left(s_{0}^{*}\right)>\right.$ $\left.z \mid Z\left(s_{0}\right)>z\right]$. Below are the resulting P-P plots.

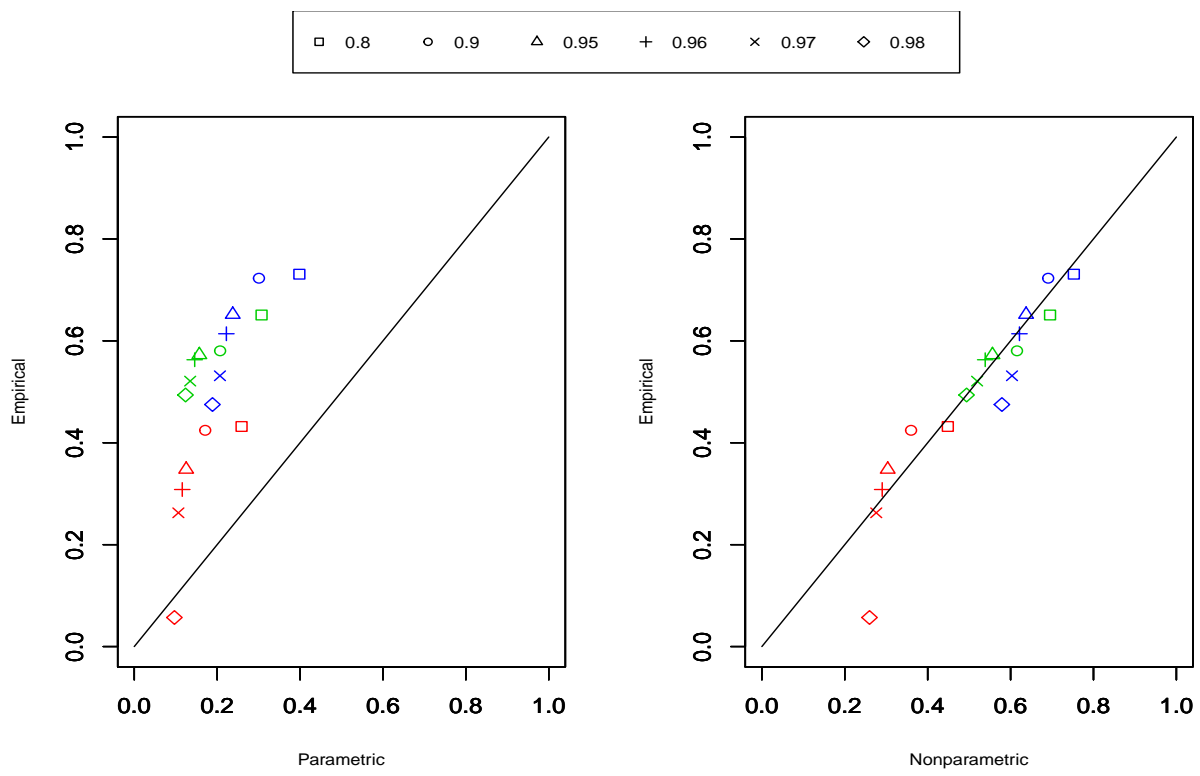

Figure 15. Diagnostic P-P plots for threshold excess conditional probabilities for the three unused sites $\{1,2,3\}$ on the map Figure 12 obtained by both approaches. The best parametric model $\mathbf{M}_{\mathbf{b}}$ as judged by the CLIC and our nonparametric approach. Green: site 1; red: site 2; blue: site 3 .

Generally, Figure 15 shows that our nonparametric approach outperforms the parametric one for predicting $\mathbb{P}\left[Z\left(s_{0}^{*}\right)>z \mid Z\left(s_{0}\right)>z\right]$. One of the justifications for this situation is that with the parametric model we have to specify a model that describes the dependence structure, and listing all choices seems to be a tedious task due to the large number of possibilities that can emerge from the $\mathrm{AD}$ and $\mathrm{AI}$ processes stemming from max-stable processes, i.e., different choices of correlation coefficient functions for the same model leads to different models. So, in this case the inaccurate choice/guess of models to be fitted may lead to severe under/over estimation of probabilities associated to simultaneous extreme events. 


\section{Conclusion}

In this paper, we have proposed a statistically efficient nonparametric model-free selection criterion. We can exploit our decision about the mixing coefficient to predict conditional probabilities of daily rainfall at unobserved sites depending on the dependence structure in the analyzed data.

The main advantage of our approach is that it is model free, unlike the parametric approach which assumes a specified model, so the risk of unaccurate choice of stochastic processes for describing the joint tail distribution may lead to severe under/over estimation of probabilities associated to simultaneous extreme events.

We have shown in our real data example that the max-mixture approach appears of interest for modeling environmental data. In particular it has the eligibility to overcome the limitations of the max-stable models in which only asymptotic dependence or exact independence can be modeled. 
Appendix A.

$a=1$

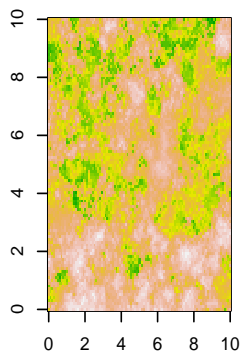

$a=3 / 4$

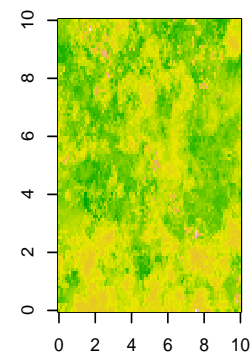

$a=3 / 4$
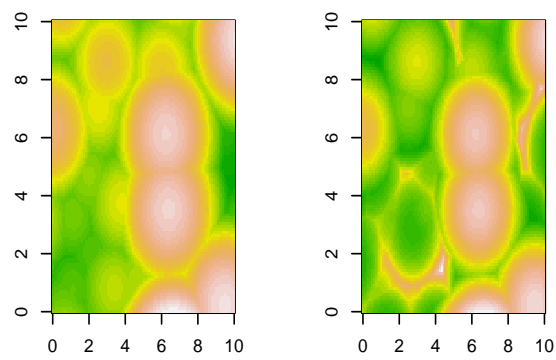

$a=1 / 2$

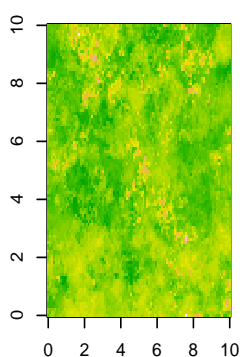

$a=1 / 2$

$a=1 / 4$

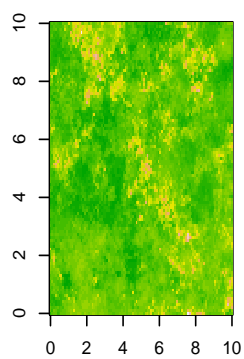

$a=1 / 4$

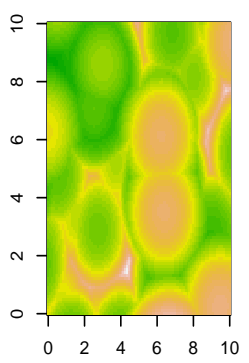

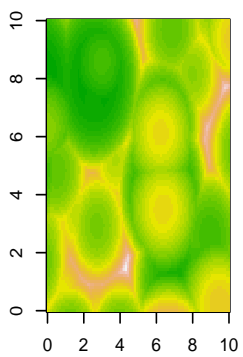

$a=0$

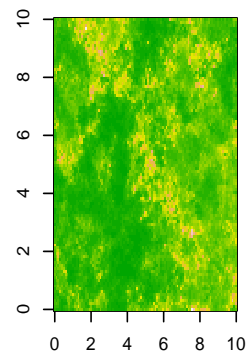

$a=0$

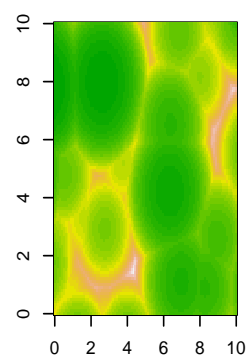

Figure 16. Simulations of the max-mixture model (2.12) on the logarithm scale according different values of mixing coefficient $a \in\{1,0.75,0.5,0.25,0\}$. Top row: $X$ is isotropic extremal- $t$ with $v_{1}=1$ degrees of freedom and $\rho(h)=\exp (-h), Y$ is isotropic inverted extremal $-t$ process with $v_{2}=2$ degrees of freedom and $\rho(h)=\exp (-h / 1.5)$. bottom row: $X$ is isotropic Brown-Resnick with variogram $2 \gamma(h)=h^{2}, X$ is isotropic inverted Brown-Resnick with variogram $2 \gamma(h)=(h / 1.5)^{2}$.
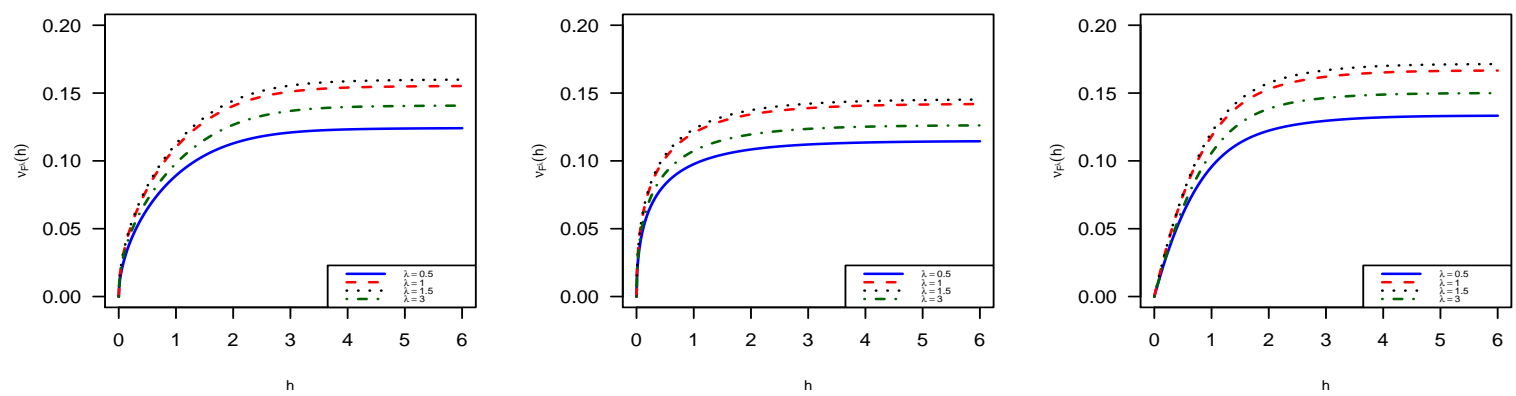

Figure 17. Theoretical $F^{\lambda}$-madogram functions 3.1 with $\lambda \in\{0.5,1,1.5,3\}$. Left panel: Max-mixture model in which in which $X$ is isotropic Brown-Resnick with variogram $2 \gamma(h)=(h / 0.5)^{2}$, and $Y$ is isotropic inverted extremal $-t$ process with $v=1$ degrees of freedom and $\rho(h)=\exp (-h / 1.5)$. Middle panel: Max-mixture model in which $X$ is isotropic extremal $-t$ with $v_{1}=1$ degrees of freedom and $\rho(h)=\exp (-h), Y$ is isotropic inverted extremal $-t$ process with $v_{2}=2$ degrees of freedom and $\rho(h)=\exp (-h / 1.5)$. Right panel: Max-mixture model in which $X$ is isotropic Brown-Resnick with variogram $2 \gamma(h)=(h / 0.2)^{2}, Y$ is isotropic inverted Brown-Resnick with variogram $2 \gamma(h)=h^{2}$.

\section{Appendix B.}

Supplementary Material related to this article can be found online at http://math.univlyon1.fr/homes-www/abuawwad/Supplementary/. 


\section{Appendix C.}

Proof of Lemma 5.1. Denoting the joint survivor function of the process $Z$ (2.12) $\mathbb{P}\left[Z\left(s_{1}\right)>\right.$ $\left.x, Z\left(s_{2}\right)>y\right]$ by $\bar{G}_{Z}(x, y)$, we may write

$$
\bar{G}_{Z}(x, y)=1-G_{X}(x)-G_{Y}(y)+G_{Z}(x, y)
$$

where $G_{X}(x), G_{Y}(y)$ are the marginal probability distribution functions of processes $X($.$) and$ $Y($.$) respectively in model (2.12) and G_{Z}(x, y)$ is the bivariate probability distribution function of the stochastic process $Z($.$) in the same model. using (2.14) and (2.16), setting x=y=z$

$$
\begin{aligned}
\mathbb{P}\left[Z\left(s_{1}\right)>z \mid Z\left(s_{2}\right)>z\right] & =\frac{\mathbb{P}\left[Z\left(s_{1}\right)>z, Z\left(s_{2}\right)>z\right]}{\mathbb{P}\left[Z\left(s_{2}\right)>z\right]} \\
& =\frac{\mathbb{P}\left(X\left(s_{1}\right)>\frac{z}{a}, X\left(s_{2}\right)>\frac{z}{a}\right) \mathbb{P}\left(Y\left(s_{1}\right)>\frac{z}{1-a}, Y\left(s_{2}\right)>\frac{z}{1-a}\right)}{1-\mathbb{P}\left[Z\left(s_{2}\right) \leq z\right]} \\
& =\frac{1-2 e^{-\frac{1}{z}}+e^{-a V_{X}(z, z)}\left\{-1+2 e^{-\frac{1-a}{z}}+e^{\left[-V_{Y}\left[\omega\left(\frac{1-a}{z}\right), \omega\left(\frac{1-a}{z}\right)\right]\right.}\right\}}{1-e^{-\frac{1}{z}}}
\end{aligned}
$$

where $\omega\left(\frac{1-a}{z}\right)=-1 / \log \left[1-e^{-\frac{1-a}{z}}\right]$, taking the advantage of the homogeneity of order -1 of the exponent measures $V_{X}($.$) and V_{Y}($.$) ; we have V_{X}(z, z)=\theta_{X} / z, e^{\left[-V_{Y}\left[\omega\left(\frac{1-a}{z}\right), \omega\left(\frac{1-a}{z}\right)\right]\right.}=$ $\left[1-e^{-\frac{1-a}{z}}\right]^{\theta_{Y}}$ and this gives (5.1).

\section{REFERENCES}

Abdul-Fattah Abu-Awwad, Vronique Maume-Deschamps, and Pierre Ribereau. Censored pairwise likelihood-based tests for mixing coefficient of spatial max-mixture models. submitted for publication, 2017 .

Manaf Ahmed, Vronique Maume-Deschamps, Pierre Ribereau, and Cline Vial. A semiparametric estimation for max-mixture spatial processes. submitted for publication, 2017.

Anestis Antoniadis, Jacques Berruyer, and René Carmona. Régression non linéaire et applications. Economica, 1992.

Jean-Noël Bacro, Carlo Gaëtan, and Gwladys Toulemonde. A flexible dependence model for spatial extremes. Journal of Statistical Planning and Inference, 172:36-52, 2016.

Liliane Bel, Jean-Noël Bacro, and Christian Lantuéjoul. Assessing extremal dependence of environmental spatial fields. Environmetrics, 19(2):163-182, 2008.

Bruce M Brown and Sidney I Resnick. Extreme values of independent stochastic processes. Journal of Applied Probability, 14(4):732-739, 1977. 
Stuart Coles, Janet Heffernan, and Jonathan Tawn. Dependence measures for extreme value analyses. Extremes, 2(4):339-365, 1999.

Dan Cooley, Philippe Naveau, and Paul Poncet. Variograms for spatial max-stable random fields. Dependence in probability and statistics, pages 373-390, 2006.

Anthony C Davison and Mehdi Mohammad Gholamrezaee. Geostatistics of extremes. 468: 581-608, 2012.

Anthony C Davison, Raphaël Huser, and Emeric Thibaud. Geostatistics of dependent and asymptotically independent extremes. Mathematical Geosciences, 45(5):511-529, 2013.

Laurens De Haan. A spectral representation for max-stable processes. The annals of probability, pages 1194-1204, 1984.

Laurens De Haan and Teresa T Pereira. Spatial extremes: Models for the stationary case. The annals of statistics, pages 146-168, 2006.

Harry Joe. Parametric families of multivariate distributions with given margins. Journal of multivariate analysis, 46(2):262-282, 1993.

Zakhar Kabluchko, Martin Schlather, and Laurens De Haan. Stationary max-stable fields associated to negative definite functions. The Annals of Probability, pages 2042-2065, 2009.

Anthony $W$ Ledford and Jonathan A Tawn. Statistics for near independence in multivariate extreme values. Biometrika, 83(1):169-187, 1996.

Anthony $W$ Ledford and Jonathan A Tawn. Modelling dependence within joint tail regions. Journal of the Royal Statistical Society: Series B (Statistical Methodology), 59(2):475-499, 1997.

Georges Matheron. Suffit-il, pour une covariance, dâêtre de type positif. Sciences de la Terre, série informatique géologique, 26:51-66, 1987.

Philippe Naveau, Armelle Guillou, Daniel Cooley, and Jean Diebolt. Modelling pairwise dependence of maxima in space. Biometrika, 96(1):1, 2009. doi: 10.1093/biomet/asp001. URL thttp://dx. doi.org/10.1093/biomet/asp001.

Thomas Opitz. Extremal t processes: Elliptical domain of attraction and a spectral representation. Journal of Multivariate Analysis, 122:409-413, 2013.

Simone A Padoan, Mathieu Ribatet, and Scott A Sisson. Likelihood-based inference for maxstable processes. Journal of the American Statistical Association, 105(489):263-277, 2010.

Mathieu Ribatet and Mohammed Sedki. Extreme value copulas and max-stable processes. Journal de la Société Française de Statistique, 154(1):138-150, 2013. 
Mathieu Ribatet, Richard Singleton, and $R$ Core Team. Spatialextremes: modelling spatial extremes. $\mathrm{R}$ package version, pages 1-8, 2011.

Martin Schlather. Models for stationary max-stable random fields. Extremes, 5(1):33-44, 2002.

Masaaki Sibuya. Bivariate extreme statistics, $i$. Annals of the Institute of Statistical Mathematics, 11(2):195-210, 1960.

Richard L Smith. Max-stable processes and spatial extremes. Unpublished manuscript, 205, 1990.

Emeric Thibaud, Raphael Mutzner, and Anthony C Davison. Threshold modeling of extreme spatial rainfall. Water resources research, 49(8):4633-4644, 2013.

Cristiano Varin and Paolo Vidoni. A note on composite likelihood inference and model selection. Biometrika, pages 519-528, 2005.

Jennifer L. Wadsworth and Jonathan A. Tawn. Dependence modelling for spatial extremes. Biometrika, 99(2):253, 2012. 